\title{
Optical and physical mapping with local finishing enables megabase-scale resolution of agronomically important regions in the wheat genome
}

Gabriel Keeble-Gagnère ${ }^{1 \dagger}$, Philippe Rigault ${ }^{2,3 \dagger}$, Josquin Tibbits ${ }^{1 \dagger}$, Raj Pasam ${ }^{1}$, Matthew Hayden ${ }^{1}$, Kerrie Forrest ${ }^{1}$, Zeev Frenkel ${ }^{4}$, Abraham Korol ${ }^{4}$, B. Emma Huang ${ }^{5}$, Colin Cavanagh ${ }^{5}$, Jen Taylor ${ }^{5}$, Michael Abrouk ${ }^{6,11}$, Andrew Sharpe ${ }^{7}$, David Konkin ${ }^{21}$, Pierre Sourdille ${ }^{8}$, Benoît Darrier ${ }^{8}$, Frédéric Choulet ${ }^{8}$, Aurélien Bernard ${ }^{8}$, Simone Rochfort ${ }^{1}$, Adam Dimech ${ }^{1}$, Nathan Watson-Haigh ${ }^{9}$, Ute Baumann ${ }^{9}$, Paul Eckermann ${ }^{9}$, Delphine Fleury ${ }^{9}$, Angela Juhasz ${ }^{10}$, Sébastien Boisvert ${ }^{2}$, Marc-Alexandre Nolin ${ }^{2}$, Jaroslav Doležel ${ }^{11}$, Hana Šimková ${ }^{11}$, Helena Toegelová ${ }^{11}$, Jan Šafár ${ }^{11}$, Ming-Cheng Luo ${ }^{12}$, Francisco Câmara ${ }^{13}$, Matthias Pfeifer ${ }^{14}$, Don Isdale ${ }^{1}$, Johan Nyström-Persson ${ }^{15}$, IWGSC ${ }^{16}$, Dal-Hoe Koo ${ }^{17}$, Matthew Tinning ${ }^{18}$, Dangqun Cui ${ }^{19}$, Zhengang $\mathrm{Ru}^{20}$ and Rudi Appels ${ }^{1,10^{*}}$ (D)

\begin{abstract}
Background: Numerous scaffold-level sequences for wheat are now being released and, in this context, we report on a strategy for improving the overall assembly to a level comparable to that of the human genome.

Results: Using chromosome 7A of wheat as a model, sequence-finished megabase-scale sections of this chromosome were established by combining a new independent assembly using a bacterial artificial chromosome (BAC)-based physical map, BAC pool paired-end sequencing, chromosome-arm-specific mate-pair sequencing and Bionano optical mapping with the International Wheat Genome Sequencing Consortium RefSeq v1.0 sequence and its underlying raw data. The combined assembly results in 18 super-scaffolds across the chromosome. The value of finished genome regions is demonstrated for two approximately $2.5 \mathrm{Mb}$ regions associated with yield and the grain quality phenotype of fructan carbohydrate grain levels. In addition, the $50 \mathrm{Mb}$ centromere region analysis incorporates cytological data highlighting the importance of non-sequence data in the assembly of this complex genome region.
\end{abstract}

Conclusions: Sufficient genome sequence information is shown to now be available for the wheat community to produce sequence-finished releases of each chromosome of the reference genome. The high-level completion identified that an array of seven fructosyl transferase genes underpins grain quality and that yield attributes are affected by five F-box-only-protein-ubiquitin ligase domain and four root-specific lipid transfer domain genes. The completed sequence also includes the centromere.

Keywords: Wheat sequence finishing, Megabase-scale integration, Optical/physical maps Grain quality, Yield

\footnotetext{
* Correspondence: rudi.appels@unimelb.edu.au

${ }^{\dagger}$ G. Keeble-Gagnère, P. Rigault and J. Tibbits contributed equally to this work.

'Agriculture Victoria Research, Department of Economic Development, Jobs,

Transport and Resources, AgriBio, Bundoora, VIC 3083, Australia

${ }^{10}$ Veterinary and Agriculture, Murdoch University, 90 South St, Murdoch,

Western Australia 6150, Australia

Full list of author information is available at the end of the article
}

(c) The Author(s). 2018 Open Access This article is distributed under the terms of the Creative Commons Attribution 4.0 International License (http://creativecommons.org/licenses/by/4.0/), which permits unrestricted use, distribution, and reproduction in any medium, provided you give appropriate credit to the original author(s) and the source, provide a link to the Creative Commons license, and indicate if changes were made. The Creative Commons Public Domain Dedication waiver (http://creativecommons.org/publicdomain/zero/1.0/) applies to the data made available in this article, unless otherwise stated. 


\section{Background}

The hexaploid wheat genome has been assembled into 21 pseudomolecules that cover more than $90 \%$ of the estimated $15.7 \mathrm{~Gb}$ of DNA that constitutes the genome [1]. Unlike previous efforts to sequence the wheat genome [2-4], the International Wheat Genome Sequencing Consortium (IWGSC) RefSeq v1.0 assembly of pseudomolecules provides a high-quality linear assembly of each chromosome from one terminal region through the centromere to the other terminal region in the form of 70-80 super-scaffolds per chromosome. Unlike advanced assemblies of human and model organisms [5], which all included sequencing of bacterial artificial chromosome (BAC)-based physical assemblies, the IWGSC RefSeq v1.0 assembly was achieved by combining a primarily whole genome short-read-based assembly with $\mathrm{Hi}-\mathrm{C}, \mathrm{BAC}$ sequencing and genetic/optical mapping information. The algorithmic advances that have made the IWGSC RefSeq v1.0 assembly possible leave a final challenge of bringing the local base-level assembly up to a finished status, where the assembly is contiguous at the megabase scale, with no gaps (Ns).

The drive for finishing the human genome has come from the requirement that all genes should be accounted for in order to establish complete coverage for functional studies [6, 7]. In the same way, a finished genome is required to understand the dynamic nature of the wheat genome $[2,8]$ and its capacity to adapt to hot and dry environments as well as very cold and wet regions, and also to capture genes responsible for traits such as yield, salinity tolerance, faster germination time or nutritional quality for fundamental and translational research. The capacity to adapt and produce grain for a variety of food and non-food products accounts for the prominent position of wheat in the modern industrial supply chain $[9,10]$. The gene space for chromosome $7 \mathrm{~A}$ was partially defined by the IWGSC Chromosome Survey Sequencing (CSS) assembly [2] and contains genes involved in determining the quality of flour (seed storage proteins, enzymes for starch and fructan synthesis, yellow pigment, preharvest sprouting tolerance) as well as many abiotic responses. Yield is widely acknowledged to be a complex trait, and components that are considered to be stable contributors to this trait include thousand kernel weight [11] and spikelets per spike [12, 13], both having significant associations with a region on 7A [13-15]. Other trait components contributing to grain yield such as grains per spike and vernalization requirements, as discussed in $[5,10]$, are also located in the same region on chromosome 7A, and together they define an important candidate target region for finishing. Another region contributing to grain quality (grain fructan content [16]) provides a second target region. In the assembly reported in the present paper, the centromere, generally considered one of the most challenging regions of the genome to assemble, was also considered using Bionano [17] maps to both confirm the assembly and to provide direction for resolving inconsistencies between cytological and assembly data. Manual annotation was performed based on the automated annotations [1] (RefSeq annotation v1.1), using alignments of available RNA-seq data $[3,18]$ to ensure gene models were consistent with transcriptome evidence.

In the present study, we used the Gydle bioinformatics (https://www.gydle.com/) software suite to produce an independent assembly of chromosome 7A which integrated a new BAC-based assembly, high-resolution genetic and Bionano map assemblies, as well as chromosome-specific mate-pair data and BAC-based physical maps. We then demonstrate the feasibility of finishing targeted regions including agronomically important regions of chromosome 7A by using the Gydle tool suite (https://www.gydle.com/) to simultaneously assess and combine our assembly with the IWGSC RefSeq v1.0 assembly in an iterative process that re-uses available raw data to resolve inconsistencies between assemblies, and between assemblies and the raw data. This approach highlights that simultaneous use of sequence and mapping resources generated by different technology platforms allows greater progress towards complete resolution of genome sequences than otherwise possible by using individual technologies. It is the first true demonstration of independent genome assembly integration that is not based on a facile merge-overlap process and provides a tractable route for finishing almost any genome region of interest in wheat, or in fact the whole wheat genome if applied universally.

\section{Results}

BAC and optical map-based assembly of chromosome 7A We assembled chromosome 7A of hexaploid wheat into 72 islands (defined below) covering a total of $752 \mathrm{Mb}$ of DNA. The assembly combined a range of data sources including a $755 \mathrm{Mb}$ physical map comprising 732 BAC contigs, represented by 11,451 BACs in 732 minimum tiling path (MTP) BAC sets, as well as mate-pair sequencing of genome-wide and chromosome-arm-specific libraries (see Methods and Additional file 1) and chromosome-arm-specific Bionano optical maps.

The islands are the combined result of scaffolding the individual BAC pool assemblies (which total $711 \mathrm{Mb}$ of sequence in 4,107 sequence contigs) using both Bionano maps (546 maps covering $746 \mathrm{Mb}$ ) and sequence alignments. The largest island covers $59.9 \mathrm{Mb}$, and $71 \%$ of the assembly is represented by 20 islands larger than $10 \mathrm{Mb}$. Our sequence assembly is highly contiguous locally with a contig mean length of $173 \mathrm{~Kb}$ and 95\% $(678.6 \mathrm{Mb})$ of its total length in 789 contigs over 100 
Kb. Very high base-level accuracy and sequence continuity was achieved through the simultaneous integration of both BAC pool and mate-pair sequencing data, physical mapping information and Bionano alignments (Fig. 1).

\section{Physical map assembly}

Flow-sorted 7AS and 7AL telosomes (Additional file 1) were used to construct BAC libraries, comprising 58,368 and 61,056 clones respectively, which were all fingerprinted as described in [19]. LTC software [20] was used to establish ordered assemblies of the BAC clones from the DNA fingerprint information in order to select an MTP BAC set for sequencing (Additional file 2: Figure S3). The following procedure was carried out for 7AS and 7AL independently: a network of "overlaps" was constructed using Sulston score cutoff $1 \mathrm{e}^{-10}$. Five iterations of increased stringency were applied in increments of $1 \mathrm{e}^{-5}$, as well as Q-clones being identified at each step [20]. BAC contigs with less than 5 clones were not included in the final outputs. The physical assembly comprised 380 BAC contigs on 7AS (352 on 7AL) and contained $42,244$ clones on $7 \mathrm{AS}$ ( 47,776 on $7 \mathrm{AL})$, from which an MTP of 5280 clones for 7AS (5832 on 7AL) was defined with an estimated total length for 7AS of $353 \mathrm{Mb}$ (402 Mb for 7AL).

\section{BAC set assemblies}

The 732 MTP BAC sets were sequenced in 813 pools, with each pool comprising no more than 40 BACs (the median number of BACs per pool was 11). This generated 1.67 billion paired reads, which were first assembled independently for each BAC set using ABySS [21] to produce a stage 1 assembly of $882 \mathrm{Mb}$ of sequence in 74,572 contigs. These contigs were used to seed a stage 2 assembly based on the use of Nuclear, Resolve and
Vision software (https://www.gydle.com/). These tools allow the sensitive alignment of raw data and resolution of conflicts with raw data, together with real-time visualization, to assemble BAC sets simultaneously using all available datasets. The datasets included the BAC set paired-end reads, mate-pair reads from whole genome and flow-sorted 7AS and 7AL telosomes and the raw data from the 7AS and 7AL survey sequencing [2]. This hybrid assembly further used physical mapping information (BAC-end derived reads identified using the cloning vector, raw fingerprinting data and $\mathrm{BAC}$ ordering) to produce assemblies consistent with the MTP layout along BAC sets and to identify and quarantine contaminant BACs for separate assembly and placement. As part of stage 3, multiple rounds of automated contig correction, extension and scaffolding, with manual curation in target regions, produced 1897 scaffolds for 7AS (2211 for $7 \mathrm{AL})$.

\section{Bionano map assembly and island construction}

Bionano optical data were generated from independently flow-sorted 7AS and 7AL telosomes producing 360,390 molecules on 7AS (416,563 on 7AL), representing 192× coverage on 7AS $(238 \times$ on $7 \mathrm{AL})$. The Bionano IrysView software was used to assemble the 178,217 7AS molecules into 783 optical maps $(145,207$ molecules into 330 maps for $7 \mathrm{AL}$ ). The total length of the optical maps was $447 \mathrm{Mb}$ for 7AS (413 Mb for 7AL) with an N50 length of $1.55 \mathrm{Mb}$ on 7AS (2.07 Mb on 7AL). These data and the BAC set stage 2 scaffolds were combined using Gydle optical mapping and assembly software to produce islands, representing connected sets of sequence scaffolds and optical maps. This process included a map validation step using molecule alignments to identify a set of high-confidence maps (272 maps on 7AS, 270 on

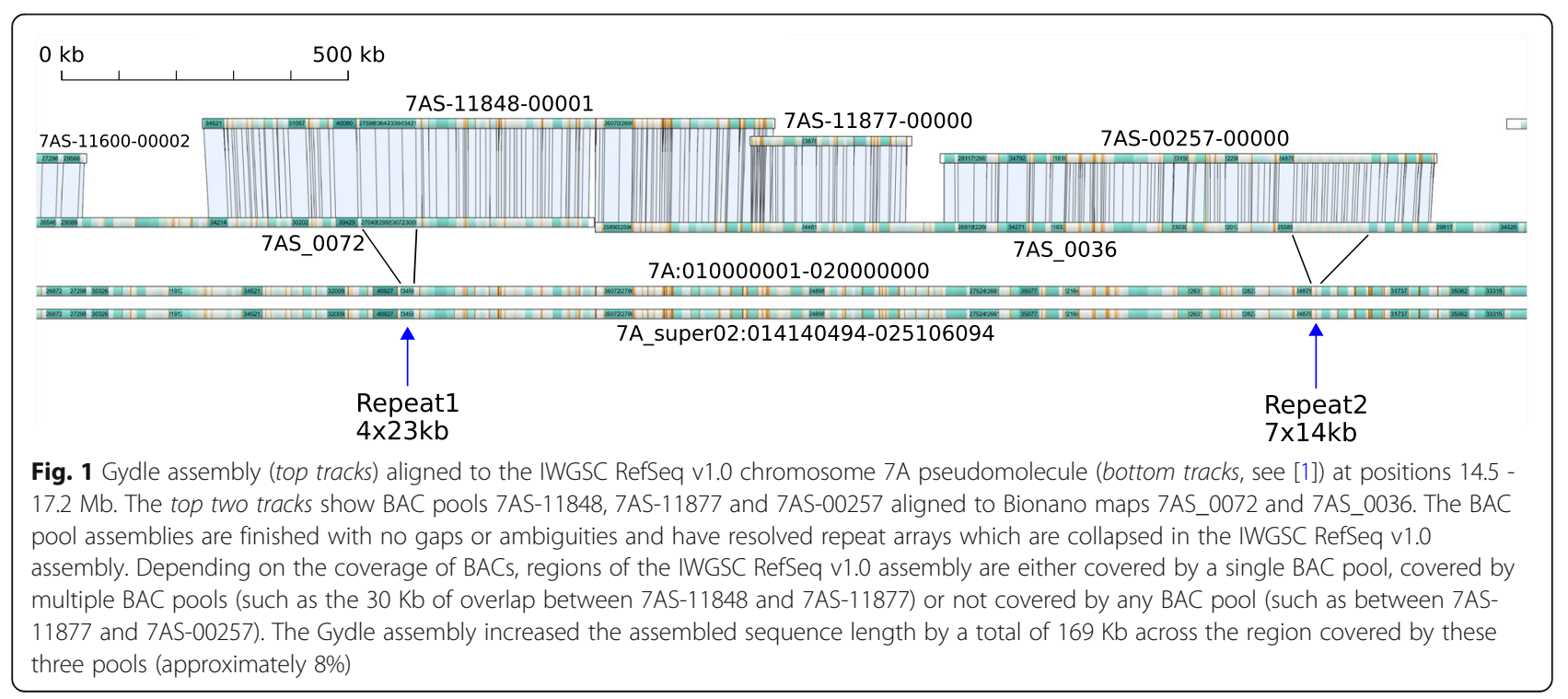


7AL), and the improvement of BAC set assemblies by using optical alignments for stitching, orienting and locally polishing scaffolds. This produced 72 final islands covering $752 \mathrm{Mb}, 711 \mathrm{Mb}$ of which was covered by BAC set sequences in 4107 contigs.

\section{BAC set finishing and assembly integration}

Several regions of the chromosome were selected for designing our finishing process (stage 3), using the Gydle software with an emphasis on complete data integration and systematic human visual review in order to achieve BAC set assembly completion: namely, a single, gapless contig of finished-quality sequence per BAC set supported by the consistency of sequence, physical mapping and optical data at the raw and assembled level, including the resolution of close repeats. We finished 30 BAC sets (representing $25 \mathrm{Mb}$ ) with this process and extended it to allow the inclusion of IWGSC (assembly and raw) data to compare, qualify and integrate the assemblies, with the view to being able to finish a sequence for the whole chromosome (i.e. outside BAC sets as well).

\section{Overview of Gydle and IWGSC RefSeq v1.0 chromosome 7A assemblies}

The IWGSC RefSeq v1.0 assembly of chromosome 7A represents $736.7 \mathrm{Mb}(\sim 90.4 \%)$ of sequence distributed relatively uniformly across the chromosome. A major strength of the IWGSC RefSeq v1.0 is the long-range organization of scaffolds and super-scaffolds into pseudomolecules. The chromosome 7A scaffolds are made up of 27,657 contigs, with a mean length of $26.2 \mathrm{~Kb}$, and $11.7 \mathrm{Mb}$ of unresolved bases $(\mathrm{N})$ in sized gaps, internal to scaffolds. Hence the IWGSC RefSeq v1.0 has a representation of most of the chromosome 7A order and arrangement, but with many small gaps internal to scaffolds and a smaller number of large gaps of unknown size between scaffolds (linkage evidence but no gap size estimation) and between super-scaffolds (no linking evidence). Our Gydle assembly represents $752 \mathrm{Mb}$ of the 7A chromosome, with $711 \mathrm{Mb}$ in near-complete assemblies of the BAC sets, which are ordered and oriented into islands with larger gaps, most of them sized, between BAC sets. Figure 1 highlights the structural differences between the assemblies, showing the near-complete representation of the underlying sequence and the concordance with Bionano optical maps within BAC pools in our assembly and the gaps between them often filled with IWGSC RefSeq v1.0 sequence. Figure 1 also highlights that in this case the Gydle assembly correctly represents the number of large tandem repeat sequences which are collapsed in the IWGSC RefSeq v1.0. These repeats are documented by Bionano maps and add about $8 \%$ to the total length of the region. This observation is consistent with the IWGSC RefSeq v1.0 wheat genome [1] which argued that much of the missing genome length in the assembly was from under-representation of arrays of repetitive sequence units.

\section{Classifying chromosome 7A into 18 connected components}

Super-scaffolds define the extent of sequences which are internally connected, ordered and in most cases oriented through underlying data links (physical or Bionano maps) without necessarily establishing the complete sequence in between or gap size. The 35 super-scaffolds of the IWGSC RefSeq v1.0 chromosome 7A pseudomolecule [1] were constructed using $\mathrm{Hi}-\mathrm{C}$ ordered scaffolds, with scaffold joins made where either the physical map via KeyGene Whole Genome Profiling (WGP $\left.{ }^{\mathrm{m}}\right)$ tags [1] or Bionano maps provided a link - a process that is sometimes prone to error due to the repetitive nature of sequences that occur at the end of scaffolds in the IWGSC RefSeq v1.0 assembly. Our island assembly integrated the physical map and Bionano data with the underlying sequence, enabling further and more accurate super-scaffolding.

Using our island assembly we were able to reduce the 35 super-scaffolds in IWGSC RefSeq v1.0 to 18. Our assembly could also orient the remaining two IWGSC RefSeq v1.0 scaffolds (of 193) that were un-oriented in chromosome 7A (7AS-00257-00000 orients scaffold138751 in minus orientation; 7AS-12029-00000 orients scaffold17971 in minus orientation). This completes the scaffold orientation across the whole of chromosome 7A. Our 18 super-scaffolds were aligned to a new high-density genetic map calculated from assigning more than 4000 markers to 900 progeny genotyped by genotyping by sequencing (GBS), from an 8-way MAGIC cross integrated with the bi-parental Chinese Spring $\times$ Renan genetic map for chromosome 7A (Fig. 2a; Additional file $3 \mathrm{a}, \mathrm{b}$ ), and this supported the overall super-scaffold order and orientation.

Using Nuclear software (see Methods and Additional file 4) to align IWGSC RefSeq v1.0 contigs (27,651 contigs; length $724.64 \mathrm{Mb}$ ) to the Gydle assembly v3.0 and stringent mapping parameters, we found 11,050 contigs that matched the Gydle assembly exactly $(193.35 \mathrm{Mb})$ and 13,539 contigs that had a partial $(>90 \%)$ sequence match $(484.54 \mathrm{Mb})$, while the remaining 3062 contigs (46.75 Mb) had no matching sequence in the Gydle assembly (consistent with the missing sequence between BAC sets). Using a stringent approach, these alignments were used to identify potential gap sequences, where only gaps between consecutive mappings of IWGSC RefSeq v1.0 contigs within Gydle contigs were selected both within scaffold and between scaffold gaps. We were able to bridge 82 of the 193 scaffold-scaffold gaps in the IWGSC RefSeq v1.0 assembly with Gydle assembly contigs spanning IWGSC v1.0 inter-scaffold gaps. Of these, 26 had a clean mapping of the flanking IWGSC RefSeq 


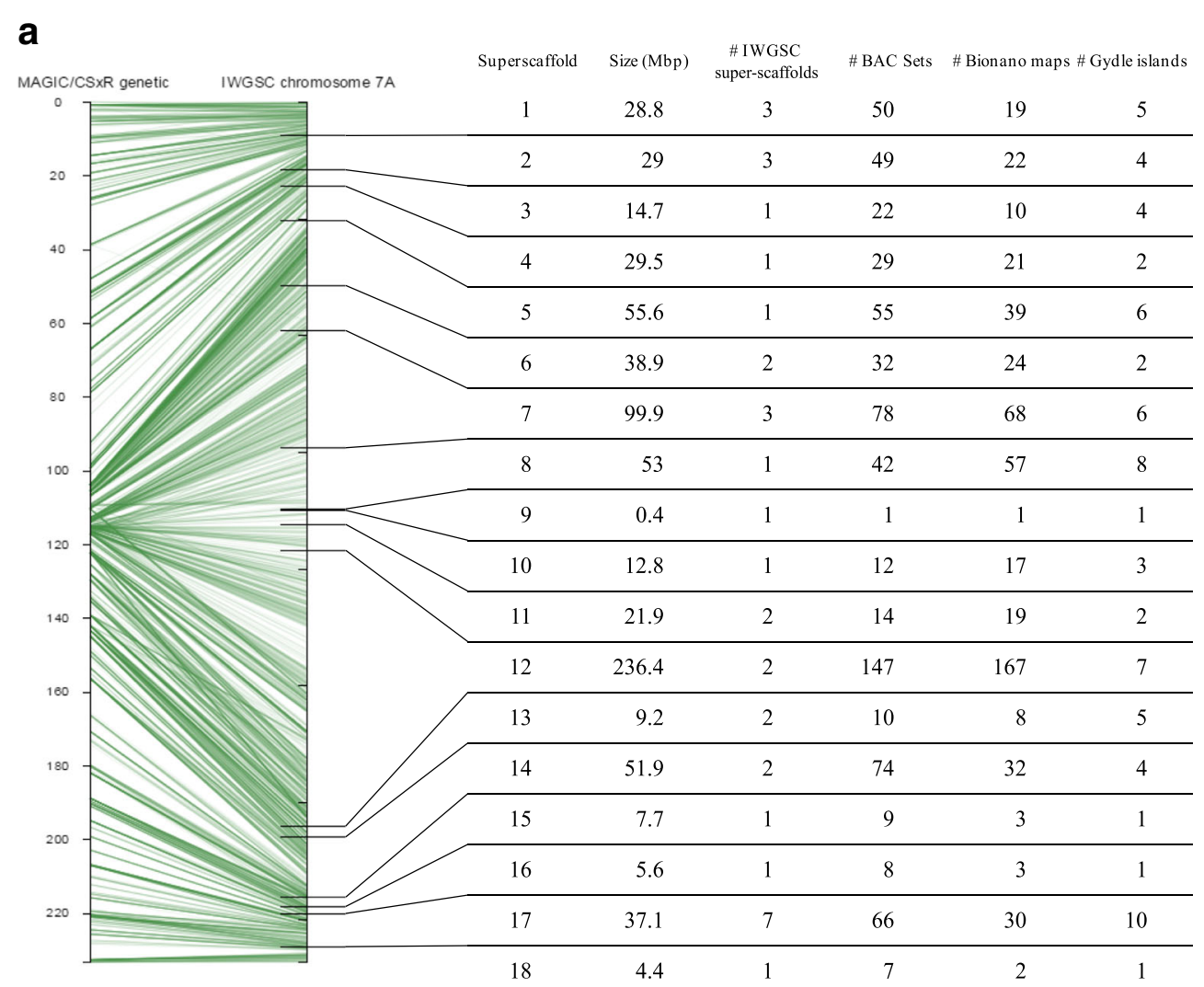

b

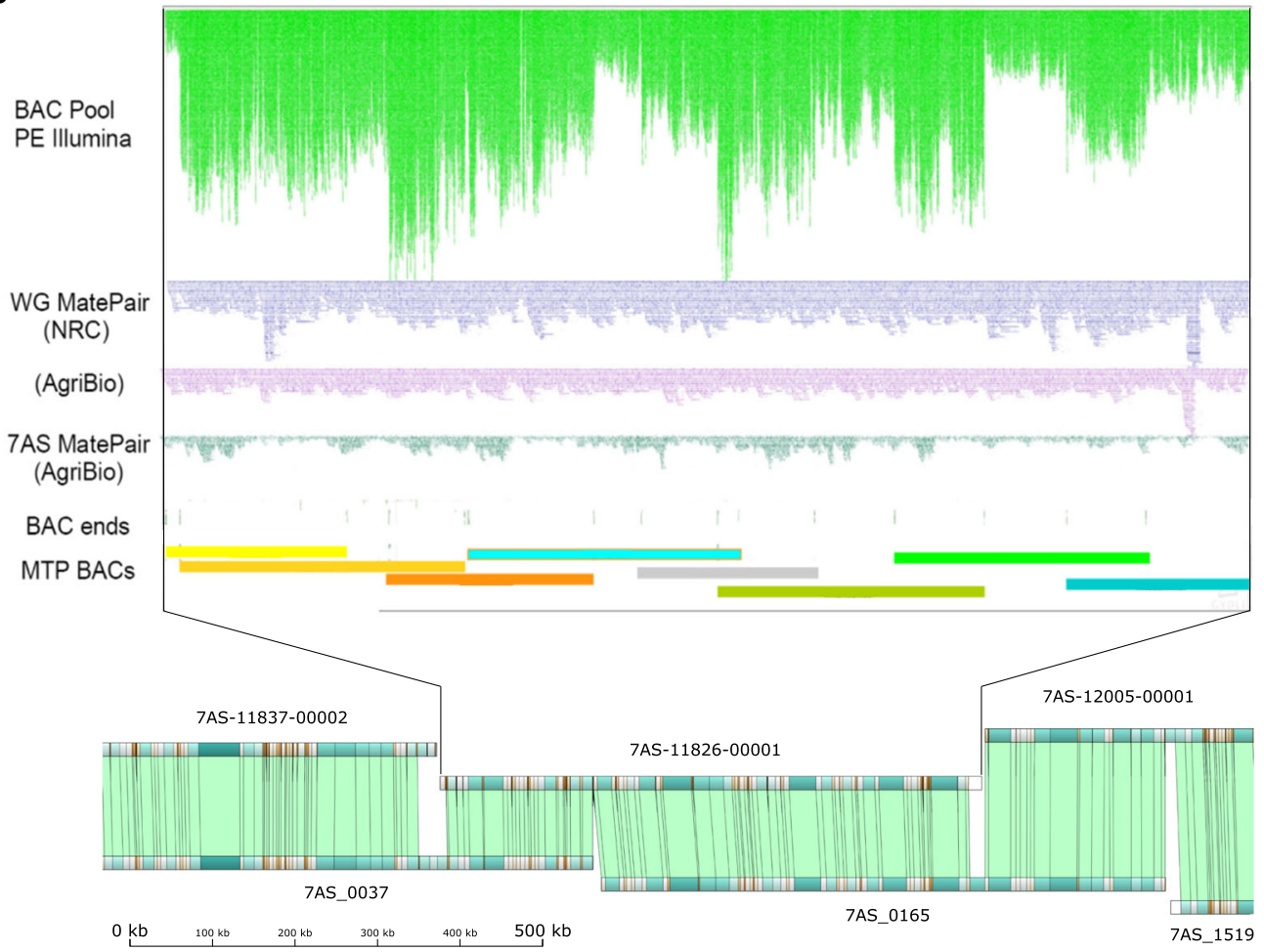

Fig. 2 (See legend on next page.) 


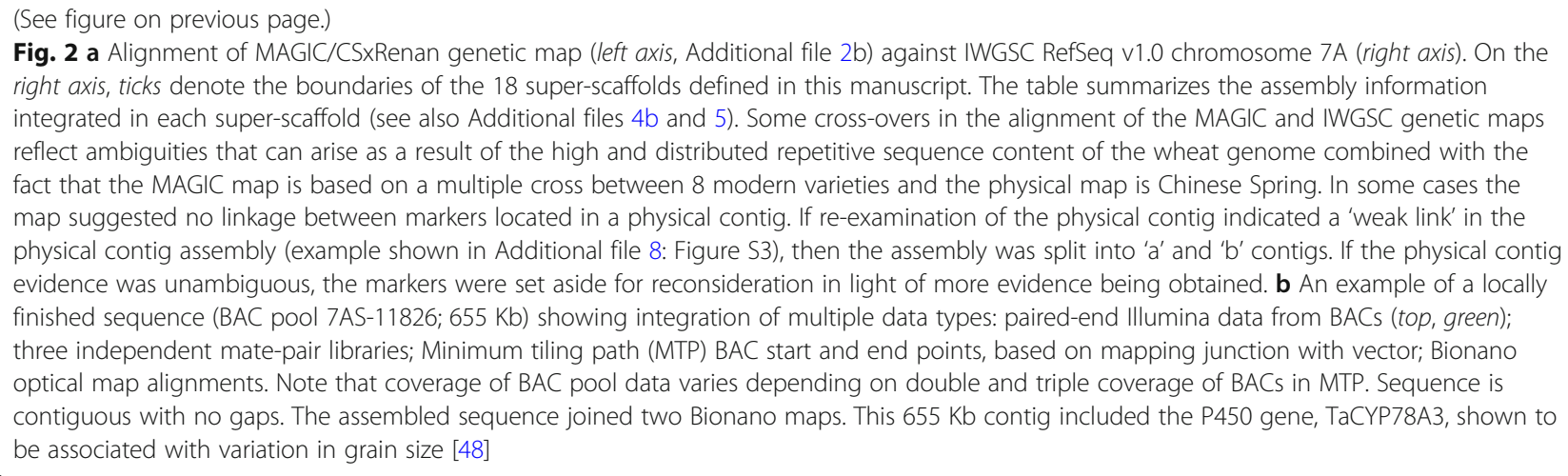

v1.0 contigs, suggesting consistency between assemblies for these regions. The reduction of 82 to 26 bridging locations reflects the a priori difficulty expected with these scaffold-scaffold sequences and our conservative approach, with the edges of scaffold assemblies in the IWGSC RefSeq v1.0 often conflicting with the Gydle assemblies. For comparison, the same analysis with the Triticum 3.0 (subsequently referred to as PacBio) assembly [4] found 88 scaffold-scaffold gaps bridged, with 54 of these in common with the Gydle set, though in only one case were the Gydle and PacBio bridging sequences the same length (Additional file 5a, b). These scaffold-scaffold gaps are clearly tractable, although they will require careful resolution, preferably combining other assembly information before bridge sequences can be determined across the wheat genome. For intra-scaffold contig-contig gaps we identified 3016 contig mappings with perfect flanking contig alignments to the Gydle assembly (Additional file 6). In total the contig-contig gap filling replaced the gap of Ns with 562,594 bp of sequence, with a mean gap size of 152.6 bp among the 2655 non-zero length gaps. The contig-contig gap sequences were observed to be generally either GC rich, often containing long homopolymer G or $\mathrm{C}$ runs, or contained di-and tri-nucleotide (and higher order) repeat sequences. Unanchored IWGSC RefSeq v1.0 scaffolds could also be assigned to chromosome 7A and accounted for 19.4 Mb of un-scaffolded sequence being identifiable as 7A against our assembly.

To assess the gene-level agreement between assemblies, we extracted the respective genome sequences (from the beginning of the 5' untranslated region (UTR) to the end of the 3' UTR) from the IWGSC RefSeq v1.0 annotation for chromosome 7A and used these to query the Gydle sequence. We found that 13,283 (96.1\%) genes were present in the Gydle assembly; of these 11,312 (81.8\%; 4370 high confidence (HC) and 6942 low confidence (LC) $(76.6 \%$ and $85.4 \%$ of their respective totals) genes matched perfectly to IWGSC sequences. Of the non-perfect matches, 414 (3\%) matched across the full length but with base-pair mismatches; 1557 (11.3\%) did not match across their full length. Across chromosome 7A we identified 107 (54 HC and $53 \mathrm{LC}$ ) genes in the IWGSC RefSeq v1.0 annotation which contained gaps (stretches of Ns) in the coding sequence (Additional file 7). Of these, 100 were complete in our Gydle sequence.

\section{Local finishing of a genome region associated with grain fructan content}

We identified a tight cluster of markers on chromosome 7A associated with grain fructan levels in a genome-wide association study (GWAS) analysis of 900 wheat lines using nuclear magnetic resonance (NMR) (3.8 ppm proton shift, see Additional file 8) and genome-wide single-nucleotide polymorphism (SNP) markers (derived from exome capture assays). The markers were contained in a single BAC contig 7AS-11582 within a $7.5 \mathrm{Mb}$ island (Fig. 3), corresponding to the IWGSC RefSeq v1.0 region spanning 3,070,713 bp to $5,459,064 \mathrm{bp}$. The 7AS-11582 contig was targeted for finishing. The tandem repeated element (four units of a $10 \mathrm{~Kb}$ repeat sequence; Bionano map, Fig. $3 \mathrm{~b}$ ), was sequenced using a single BAC (7AS-066B03) covering that repeat and PacBio sequencing combined with short-read Illumina data, physical mapping and optical data during the finishing process.

Through iterative rounds of automated and manual assembly, we constructed a final sequence assembly, integrating and consistent with all available raw data sources, of 2,397,551 bp in a single contig with no gaps or unresolved bases. The IWGSC RefSeq v1.0 sequence comprised 3 scaffolds and 105 internal gaps (giving a total of 107 gaps across the region, Additional file 2: Figure S5). Of these, 98 were filled with sequences from the Gydle assembly, with a common observation that the gap sequences were either GC rich (12 gaps with $80 \%+$ GC) and/or contained a homopolymer run of G10 + or $\mathrm{C} 10+$ (52 gaps). Illumina sequencing technologies are known to have difficulties in regions with G/C homopolymer runs [22] and, while the coverage in these regions is generally lower than that of surrounding sequences, supporting raw data for these missing 


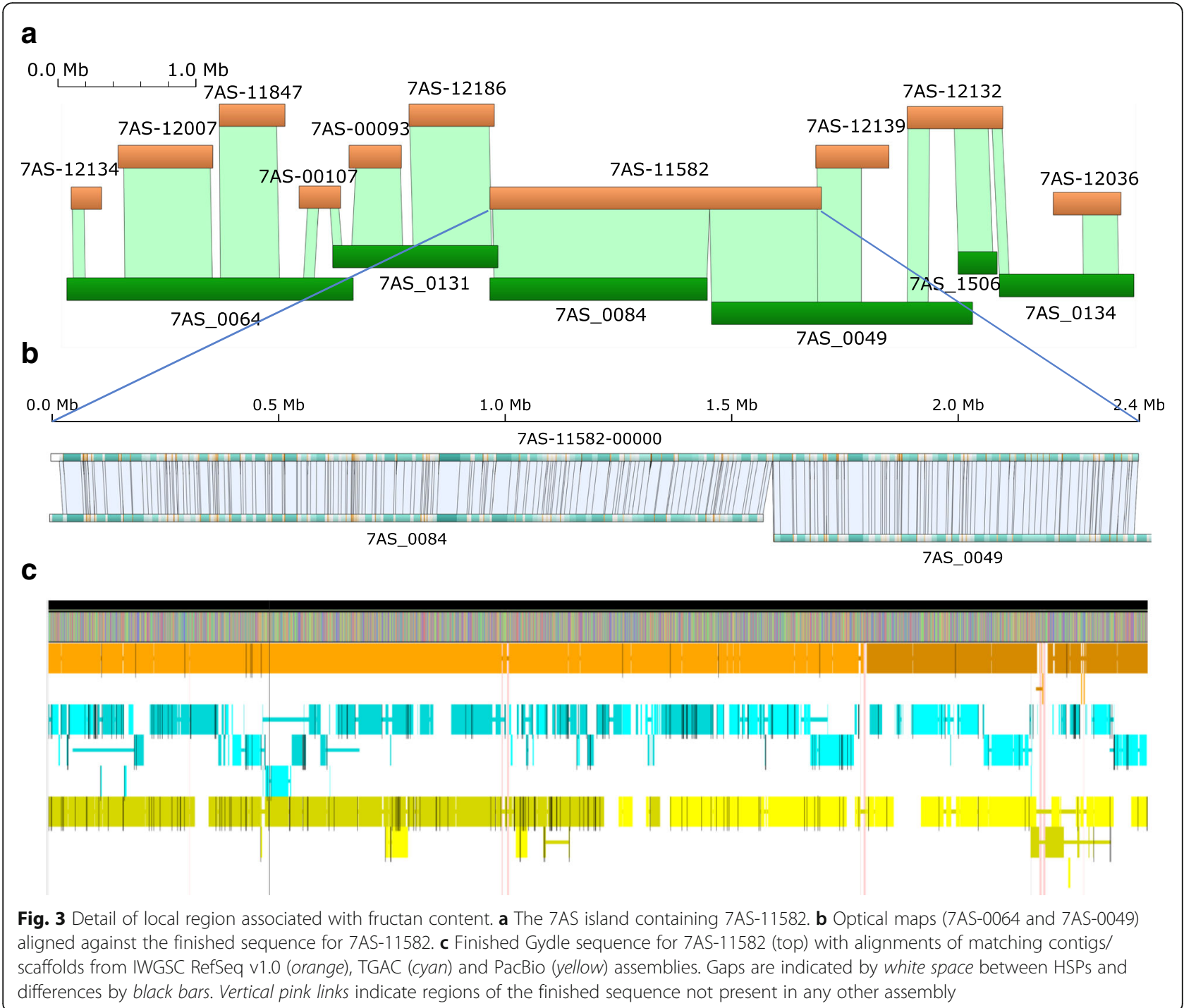

sequences are often present in existing datasets. The longest filled gap sequence in the 7AS-11582 region was $6826 \mathrm{bp}$ with a mean filled gap size of $306 \mathrm{bp}$ (median $92 \mathrm{bp}$ ). The remaining seven gaps were closed with either short sequence overlaps between neighbouring contigs or subtle rearrangements of the final sequence versus the original contig order. A small number of within-contig insertions (eight) and deletions (nine) were also found. The majority of these were single bases, and three were greater than $100 \mathrm{bp}$.

We identified scaffolds from The Genome Analysis Centre (TGAC) [3], PacBio [4] and IWGSC RefSeq v1.0 [1] assemblies using our finished sequence as bait and applying the same alignment parameters for each. Figure 3c summarizes these assemblies aligned against the finished Gydle 7AS-11582 sequence. As expected, no assembly fully represented the entire region, and all assemblies were fragmented around the repeats, highlighting their difficulty for assembly. Comparison of assembly completeness and similarity across this region indicates that assembly merging as a means of genome finishing will require a careful strategy capable of deciding between competing options. Clearly, simple merge-overlap approaches are not likely to improve the entire genome representation provided in the IWGSC RefSeq v1.0, and an approach that re-references the raw data (preferably from multiple sources simultaneously) to resolve inconsistencies will be required.

One of the most important attributes of having a locally finished sequence is the impact on the accuracy of the gene annotation. There were $62 \mathrm{HC}$ and $68 \mathrm{LC}$ genes annotated across the 7AS-11582 region. Five of the $\mathrm{HC}$ genes had gaps within the genomic sequence and, of these, two (TraesCS7A01G010500 and TraesCS7A01G010800) had gaps within their coding sequence (CDS). The finished assembly completed these genes 
and enabled the gene models to be updated. For Traes $C$ $S 7 A 01 G 010500$ the gene model was incomplete in all other available annotations of wheat, and the finished gene model was found to be a novel variant of a BAG family molecular chaperonin regulator seven gene (UniProtKB - Q9LVAO (BAG7_ARATH)). Close proximal regions to genes generally harbor functional elements, and the finishing process in these regions closed 38 (18 HC; $20 \mathrm{LC}$ ) gaps within $5 \mathrm{~Kb}$ of annotated genes. Of particular interest for grain quality was the identification, confirmed through the manual curation of the gene models across the finished sequence, of a tandem array of seven glycoside hydrolases (EC 3.2.1, labelled a to g), including the gene model GH32b being assigned as a 1-FFT (fruc$\tan 1$-fructosyltransferase) on a sequence similarity basis and GH32g being assigned to 6-SFT (sucrose:fructan 6-fructosyltransferase). Both these genes are expressed in the grain and stem, based on alignments of RNA-seq data from [18], and represent good candidate genes for variation in grain fructan levels.

\section{Local finishing of a genome region associated with grain number and weight}

Published studies have mapped yield quantitative trait loci (QTL) to the long arm of chromosome 7A with varying degrees of resolution [23]. Using a RAC875 $\times$ Kukri cross, we mapped yield and two yield components, thousand kernel weight (TKW) and kernels per spikelet across the length of chromosome 7A (Additional files 8 and 9). A cluster of four TKW QTL was in the 172.4-177.0 cM region of the RAC875/Kukri map (Additional file 9). These co-located with the QTL TaTKW-7AL which was mapped to a $1.33 \mathrm{cM}$ interval on chromosome 7A (between 90K SNPs IWB13913 and IWA5913 [15]) and a QTL for spikelet number per spike [13] in the same interval. These QTL define a core yield QTL region located between 672,014,054 bp and $674,276,807$ bp in the IWGSC RefSeq v1.0 7A pseudomolecule, which we targeted for complete sequence finishing (Fig. 4). The region is covered by two scaffolds (scaffold274331-1 and scaffold91613) in IWGSC RefSeq v1.0 [1], where the $2.262 \mathrm{Mb}$ pseudomolecule sequence contains 37,065 uncalled bases $(\mathrm{N})$ in 101 gaps. In the Gydle assembly, the core region, contained within a single island, was covered at $94 \%$ by 5 non-overlapping BAC sets (7AL-12138, 7AL-05057, 7AL-12241, 7AL-00419 and 7AL-11456). We performed finishing on these BAC sets to produce 2,125,896 bp of the region, then finished the intervals between BAC sets using the raw sequence data (IWGSC and our 7A mate-pair libraries) combined with Bionano to resolve 144,424 bp. The finished core yield QTL region is a gapless contig of 2,270,131 bp (Additional file 10).

Manual curation of all the IWGSC gene models across this region enabled many small annotation inconsistencies to be detected and corrected, most of which arose because of micro-assembly ambiguities. Across the QTL core region there were 61 genes (27 $\mathrm{HC}$ and $34 \mathrm{LC}$ ) annotated in IWGSC RefSeq v1.0 of which 6 had gaps within their genomic sequence in the original assembly (Additional file 11). The sequence downstream of the core QTL $(674,273,097$ to $674,876,866 \mathrm{bp})$ contained 27 annotated genes $(12 \mathrm{HC}$ and $15 \mathrm{LC})$ which included a cluster of 8 Hydrophobic-domain protein family genes ([1], cortical cell delineating class, specifically expressed in roots). We used the available finished sequences to investigate linkage disequilibrium (LD) across the QTL region in 863 unrelated wheat accessions each assayed with Roche exome capture technology (Fig. 4; Additional file 8). Seven blocks of high $\mathrm{LD}$ are seen across the region and clearly define targets for further fine mapping of the TKW and kernels per spikelet in blocks 2-5 (Fig. 4). The gene function predictions based on the domains in the translated protein sequences (Additional file 11) serve to further refine a candidate gene list.

\section{Multiple windows into the wheat chromosome 7A centromere}

Centromeres mediate chromosome attachment to microtubules and ensure proper segregation of the sister chromatids during mitosis and meiosis [24]. While the active centromere and associated kinetochore complex is characterized in plants by the location of CENH3 binding sequences [25], various working definitions include reduced recombination rates, methylation patterns, transposable element repeat patterns and constitution and chromosomal centromere breakpoints. Taking a classical definition of the centromere as the region of suppressed recombination, we defined a centromere region in chromosome 7A based on an analysis of more than 900 lines in an 8-way MAGIC population cross, genotyped with a targeted GBS assay (Additional file 3), and determining the parental donor of chromosome segments for counting cross-overs. The centromere region defined by suppressed recombination spans almost half the chromosome, between approximately $175 \mathrm{Mb}-600 \mathrm{Mb}(425 \mathrm{Mb})$. Within this region a $170 \mathrm{Mb}$ (spanning 270-440 $\mathrm{Mb}$ ) region of no cross-overs containing a smaller $60 \mathrm{Mb}$ region (spanning 320-380 Mb) enriched for centromere-specific centromere retrotransposon for wheat (CRW) (Cereba/Quinta) repeat families was identified (Fig. 5a).

Alignment and anchoring of the broad centromere region defined by the CRW sequences to the rice chromosome 8 functional centromere region (Fig. 5b) identified six highly conserved genes (TraesCS7A01G284500, TraesCS7A01G28 8700, TraesCS7A01G294900, TraesCS7A01G295300, Traes CS7A01G295400, TraesCS7A01G292400) that spanned both the 7A and rice 8 centromere regions and were homologs to the rice genes identified by Yan et al. [26] as highly conserved across crop plants. Manual annotation of 7A 


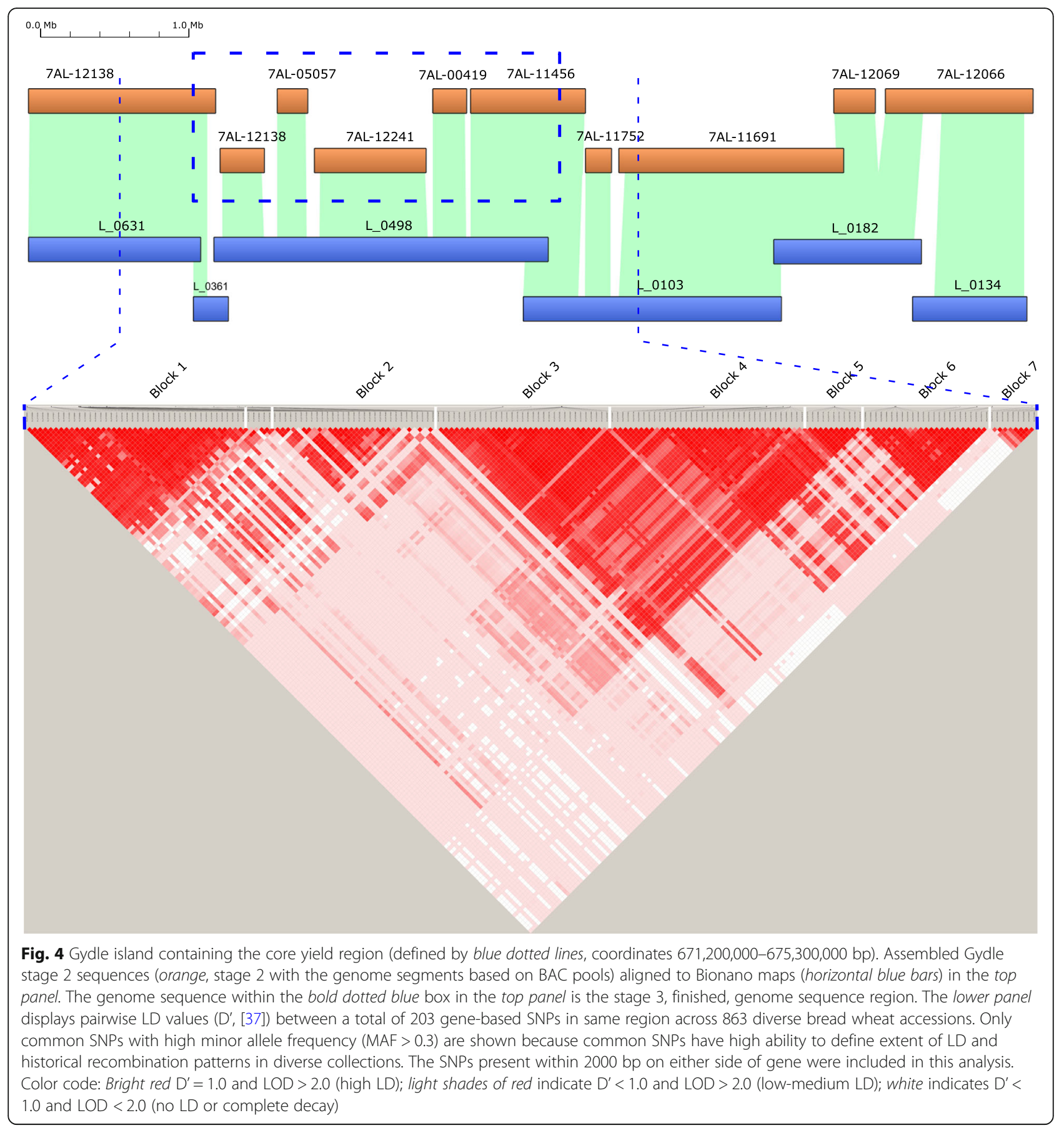

genes following gap closure allowed the functional domain of the 7A centromere to be defined through synteny alignment (Fig. 5b, Additional file 12) to the rice chromosome 8 centromere.

Complete agreement between separate 7AS and 7AL telosome assemblies and data (raw flow-sorted chromosome paired-end read data [2], Gydle BAC sets and Bionano maps sequences) provided additional evidence for the location of a core region of the 7A centromere, with a $5 \mathrm{Mb}$ region of overlap between the two telosomes resulting from asymmetrical positioning of the breakpoints (Figs. 5a and 6). At the end of the 7AL telosome, evidence from the Bionano map indicated that the terminal $50 \mathrm{~Kb}$ had been duplicated (in reverse complement) on the 7AL telosome, with this extended sequence not appearing in the 7AS side of the assembly. Coverage of raw 7AL read data across the IWGSC RefSeq v1.0 chromosome 7A centromere supports the presence of this sequence duplication at this end of the 7AL centromere (Fig. 6d, increased read coverage at centromere end of 7AL 


\section{a}
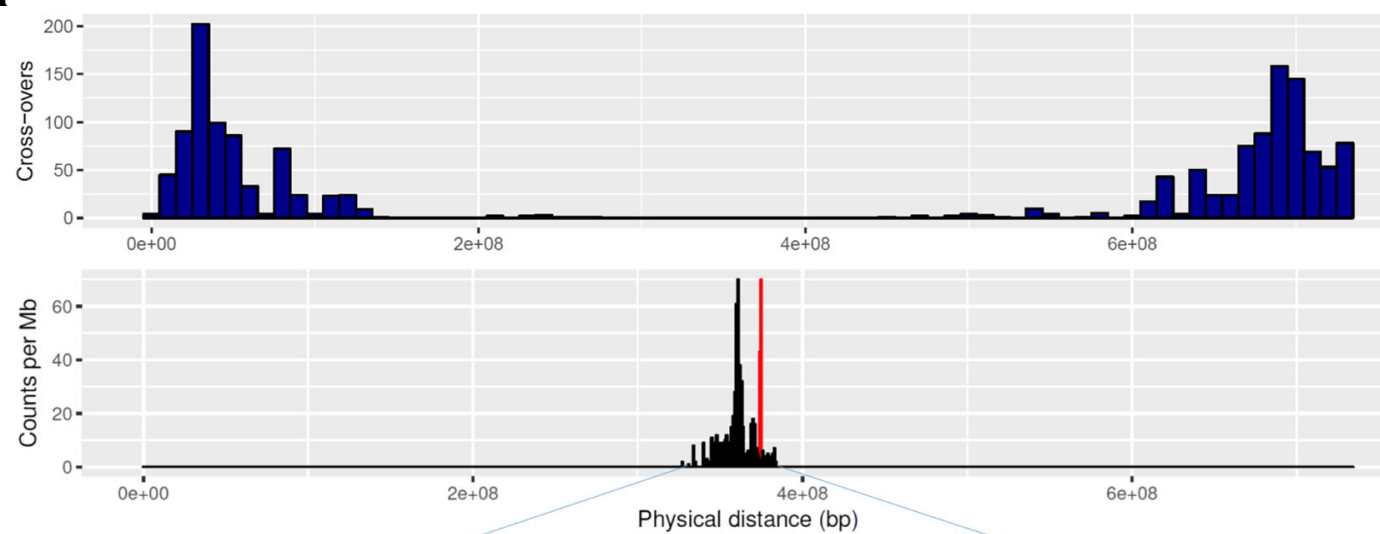

end of 7AL telosome end of 7AS telosome

7AS/7AL overlap

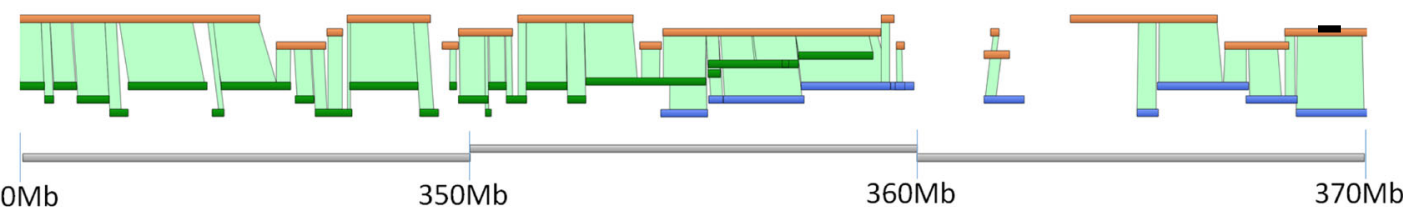

b

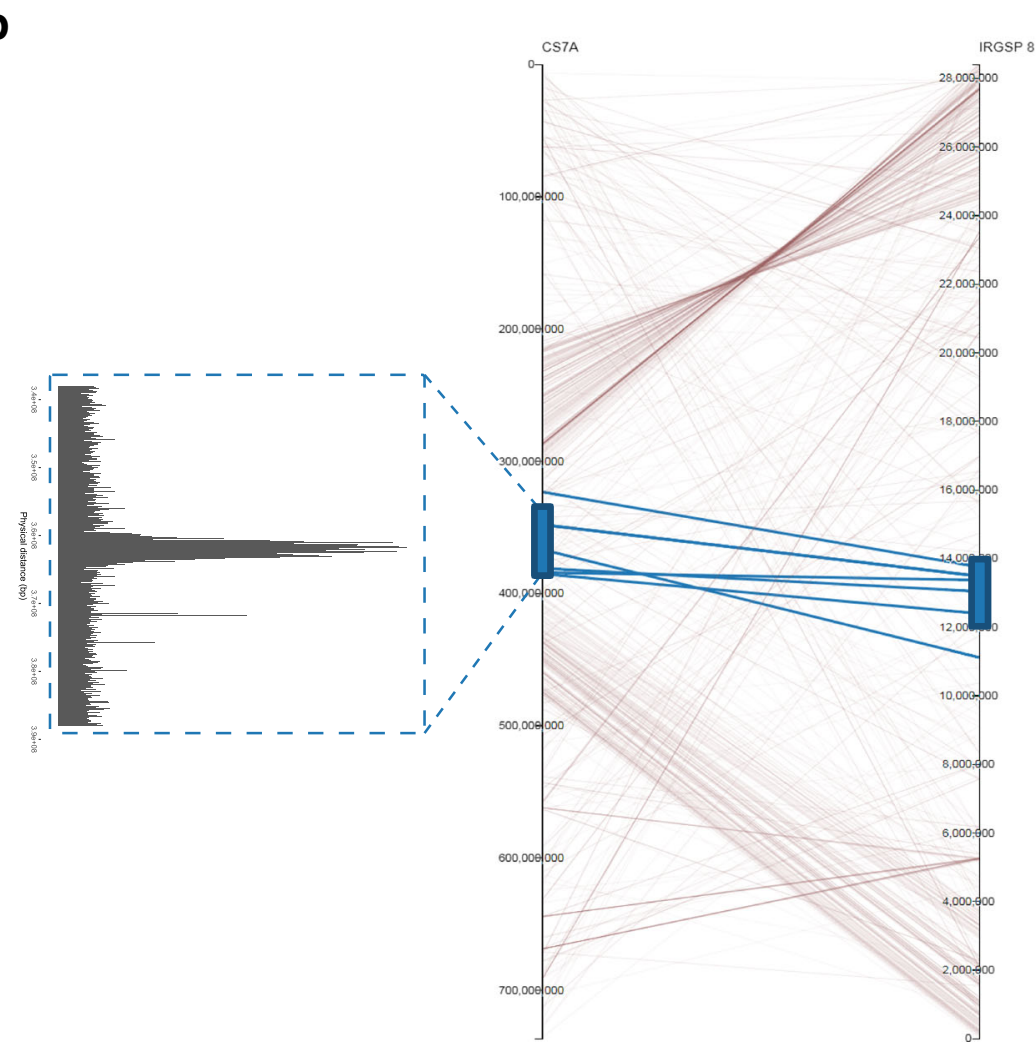

Fig. 5 (See legend on next page.) 
(See figure on previous page.)

Fig. 5 a The 7A centromere. The top panel shows cross-over counts from an analysis of 900 lines (only cross-overs from 465 lines shown; see Additional file 1) of a MAGIC population (10 Mb bin size) across the entire chromosome and identifies a region of zero recombination traditionally associated with the centromere. The second panel shows this region is the primary location of the Cereba TEs that define wheat centromeres. Within this region we also identified a compact cluster of Tai 1 sequence elements shown in red. The third panel indicates the location of the breakpoints that generated the 7AS and 7AL telosomes, and the bottom panel shows the Gydle islands (sequences in orange) and Bionano maps (7AS in green, 7AL in blue) for this region tiling the IWGSC RefSeq v1.0 (gray) from $340 \mathrm{Mb}$ to $370 \mathrm{Mb}$. The break in both the Gydle and Bionano maps in the $349 \mathrm{Mb}$ region is referenced in the text as well as Fig. 6a as a possible location of CENH3 binding sites. $\mathbf{b}$ The 7A centromere aligned to rice chromosome 8. Lines indicate syntenic genes, with conserved gene models between the two centromere regions highlighted in blue. Equivalent locations of the CENH3 binding sequences shown on the right and left sides. The CENH3 plot for the rice 8 centromere (right side) was modified from Yan et al. [26]

indicated by a dotted blue box); the duplication is absent from a standard chromosome 7A.

The active centromere and associated kinetochore complex in plants can also be defined based on the location of the CENH3 binding domain [27]. We aligned CENH3 ChIP-seq data for wheat $[25,27]$ to the IWGSC RefSeq v1.0 and found a $5 \mathrm{Mb}$ region on the proximal side of 7AL to the breakpoints (not in the region of overlap between the 7AS and 7AL assemblies) as the primary source of similarity to the CENH3 binding sequences and CRW repeat sequence families (Fig. 6a). This located the main CENH3 binding domain represented in the assembly to be on 7AL. Analysis of an independent assembly of Chinese variety Aikang 58 showed the same chromosomal structure, although the size of the core CENH3 binding/CRW repeat region was larger. Tiling of

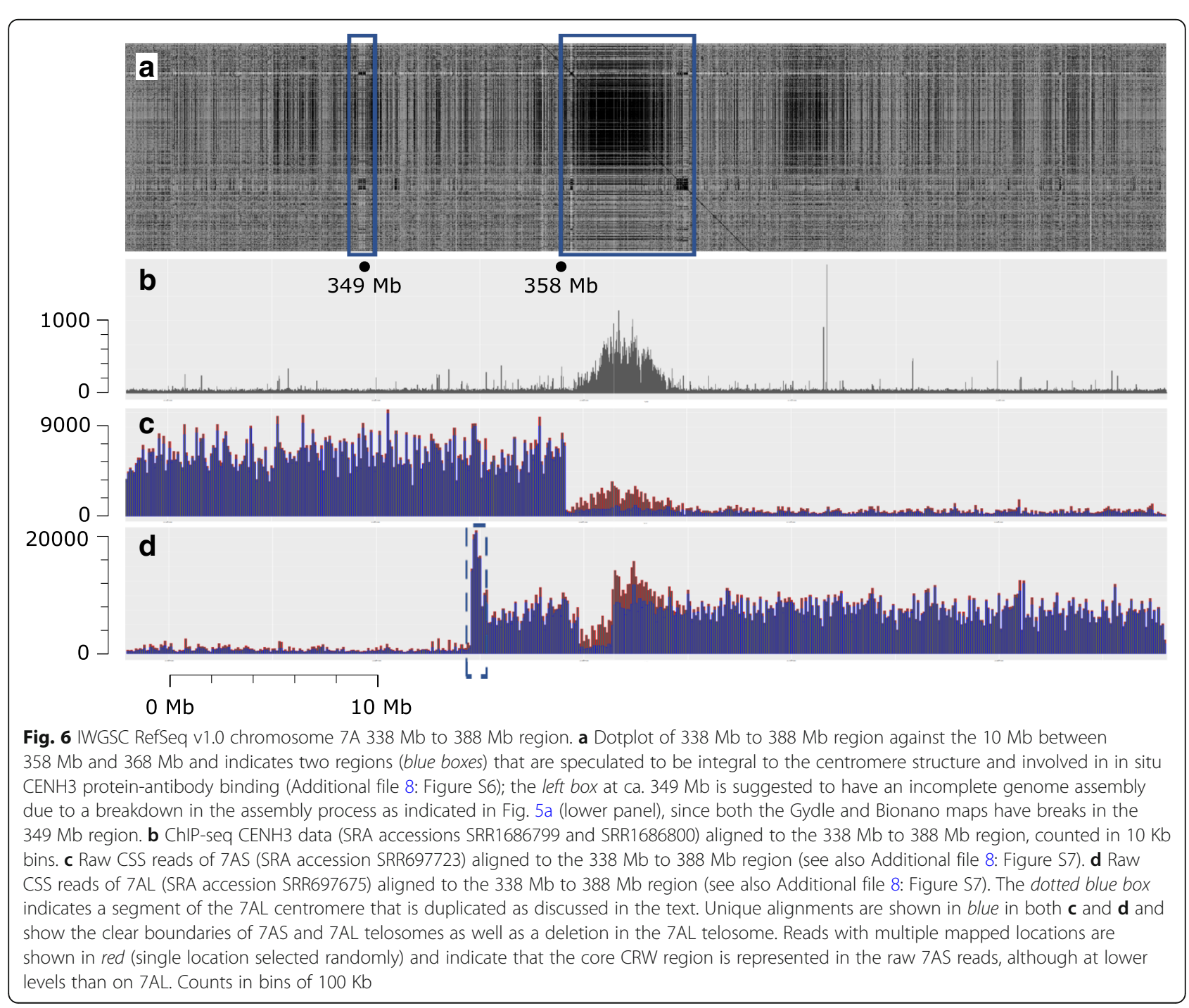


the Gydle assembly around this region in IWGSC RefSeq v1.0 identified a gap in coverage of BAC data on the 7AL side of the assembly (Fig. 5a). Alignment of raw IWGSC CSS data across the region (Fig. 6c, d) showed a sharp drop in coverage to background levels at exactly the same location; however, alignment of the raw data used in the IWGSC RefSeq v1.0 assembly indicated this region was present in the whole genome data (Additional file 2: Figure S7), implying a deletion of around $2 \mathrm{Mb}$ in the 7AL ditelosomic stock (Fig. 6).

Although the 7AS telosome appears to be missing a major CENH3 binding domain, records for tracking the transmission of the two telosomes in the Wheat Genetics Resource Center, Kansas State University showed the transmission rates for the two telosomes were similar, implying that 7AS must also have an active centromere. We used the in situ localization of the CENH3 antibody (Additional file 2: Figure S7, Additional file 13, [28]) to show that the 7AS telosome also has a localized CENH3 binding domain near the telosome breakpoint. Both telosomes carried a similar level of CENH3 antibody binding protein in the centromere regions, based on the analysis chromosome spreads shown in Additional file 2: Figure S7, suggesting it unlikely that there exists a major difference in genome structure of the centromere. Furthermore, we found evidence that sequences from the CRW region of this scaffold were present in the 7AS telosome at low levels (Fig. 6a). Close inspection of the tiling of the Gydle sequence around scaffold96327 (a single scaffold unconnected to the surrounding scaffolds in the pseudomolecule, also a single island in the Gydle assembly) found highly dense copies of Byron CACTA elements (as well as representative copies of other CRW elements, Fig. $6 \mathrm{a}$ at position $349 \mathrm{Mb}$ ) and suggests this as a possible location for the 7AS CENH3 binding domain sequence within the 7A functional centromere region analogous to that found in rice centromere 8 .

\section{Discussion}

In this manuscript the resources for finishing a wheat reference genome sequence were defined at two levels, namely, micro-scale and macro-scale. At the macro-scale the IWGSC RefSeq v1.0 assembly provided a pseudomolecule against which our independent BAC-based assembly could be aligned, enabling a reduction in super-scaffold number, the completion of super-scaffold ordering and orientations and the local solving of micro-scale inconsistencies and deletions. This capacity enabled, across the whole chromosome, 52 CDSs in the IWGSC RefSeq v1.0 to have sections of Ns filled and gene models updated. In target regions, a method that combines multiple resources such as the raw Chromosome Survey Sequencing (CSS) [2], high-density molecular genetic maps ([29], Additional file 3) and Bionano maps was able to produce a finished sequence (Methods, Additional file 4). The Bionano maps were particularly valuable as an independent source of linear sequence information when assemblies conflicted. Two target regions of chromosome 7A were studied in detail to explore the requirements for finishing the genome sequence of the reference assembly at a broader level. These sequences are the largest complete sequences available in wheat and highlight that merging sequences from multiple assemblies to achieve complete finishing is possible but will require the re-referencing, preferably simultaneously rather than sequentially, of the multiple raw datasets and types to provide final validation where assembles agree, and to provide information to resolve conflicts between assemblies as these are found.

One of the $2.5 \mathrm{Mb}$ regions that was finished overlapped the QTL initially defined by Huynh et al. [16] for fructan content in the grain. In our analysis this region was shown to contain a tandem array of seven glycoside hydrolases (EC 3.2.1, labelled a to g) that were of particular interest since the gene model GH32b could be assigned to 1-FFT on sequence similarity basis and GH32g to 6-SFT. Both these genes are key in the fructan biosynthetic pathway [30]. The GH32 genes were expressed in the grain and stem and the analysis of variation in grain fructan levels from 900 wheat lines characterized using exome capture indicated that more than half of the SNP variation in the QTL region associated with variation in grain fructan levels located to the GH32 family genes. For the homoeologous GH32 array on chromosome 7D, the most highly significant association across the entire genome was also in this region, and it is thus evident that selection at multiple loci is required for a phenotype such as grain fructan levels.

The region associated with TKW and spikelet number, within the broad yield QTL region on 7AL, required a more extensive integration of the IWGSC RefSeq v1.0 and Gydle assemblies. Solving the complete sequence for this region showed that distinct linkage blocks existed in diverse worldwide wheat lines, indicating that fine mapping this region through association analysis will be challenging. The gene families within linkage blocks included repetitive gene models annotated as housing domains involved in apoptosis as well as root morphology; they thus provide targets for establishing a framework for strategies to select for variation which includes variation in copy number, as suggested in [1].

The centromeres of chromosomes have been studied extensively [31] in microorganisms, animals and plants. The centromere of 7A was located within the $\mathrm{C}$ region [1] in chromosome 7A extending from position 240 to $410 \mathrm{Mb}(170 \mathrm{Mb},[1])$ and could be further defined as a 58 $\mathrm{Mb}$ region based on the presence of the reverse transcriptase sequence from the Cereba element (AY040832). Except for one unit located at $67 \mathrm{Mb}$ in the telomeric region 
of 7AS, the Cereba element was unique to the centromere region within 7A. The detailed structure studies indicated that at least two domains for centromere activity existed within the functional domain that was syntenic to the rice chromosome 8 centromere. The centromere region contained 62 genes, and 5 of these genes were also located in the rice chromosome 8 centromere and provided the basis for defining a syntenic functional centromere. Although the CENH3 binding sequences on 7AS were not as clearly defined as in 7AL, we speculate that this is due to a breakdown of the assembly process in the respective region (349 Mb region, see Figs. 5a and 6a). The available data suggest the reduced CENH3 protein-antibody binding assayed in both the 7AS and 7AL telosomes (relative to the level of binding to normal chromosomes Additional file 2: Figure S6) is sufficient for the retention of centromeric activity. The analysis also indicated that the terminus of the 7AL centromere had a terminal $50 \mathrm{~Kb} \mathrm{du}-$ plication of a sequence that is located between the two proposed CENH3 protein-antibody binding domains. In addition, an element, Tai l (AB016967) [32] was found to have 100 units in the region $374.7-376.9 \mathrm{Mb}$ (on 7AL) and is unrelated to Cereba or the rice/maize centromere repeats but exists within the Quinta retrotransposable element. In situ hybridization [32] shows Tai 1 is centromeric to all wheat chromosomes. The incursion of this most recent transposable element (Quinta/Tai l) is a striking feature here in that the Tai 1 sequence is a dispersed repeat in grasses related to wheat and is consistent with it being a recent addition to the wheat genome that has not had enough time to spread more widely. It is possible that new clusters of repetitive elements significantly enhance the network of interactions in which the centromere is involved in meiosis and mitosis [33].

\section{Conclusions}

Chromosome 7A provided a useful model to carry out analyses that establish a foundation for developing an advanced, version 2.0, high-quality wheat reference genome assembly. The strategy developed in the present manuscript indicates that the required assembly algorithms and sequence data exist, while future investment in long-read data, such as Bionano optical maps, will provide the complete resources necessary for integration of raw data into well-developed templates of the wheat reference genome, sufficient for the accurate interpretation of sequences from new wheat varieties. The suites of genes identified in regions of the genome associated with grain yield and quality provide a basis for identifying gene family copy number variation and new molecular markers for the rapid selection of difficult phenotypes in breeding programs. A key utility of the IWGSC RefSeq v1.0 genome assembly [1] is to accelerate QTL mapping and then support the gene cloning or perfect marker identification process in both fundamental and translational research. At the back end of these processes, it is the genome assembly quality that most often inhibits progress. Likewise, the use of gene editing and other similar modern breeding methods requires base-level accuracy in focus regions. Importantly, the finished regions described in this research span the flanking markers of known QTL, and hence these regions can be studied in full without unknown assembly issues impeding progress.

\section{Methods \\ Independent assembly of chromosome 7A}

The BAC library of 119,424 BACs $(58,368$ and 61,056 on 7AS and 7AL, respectively) from flow-sorted chromosome arm 7A DNA was fingerprinted using the SNapShot method [19] and assembled into physical contigs using LTC software [20]. The physical map comprised 732 BAC contigs and an MTP of 11,451 BACs totalling an estimated $755 \mathrm{Mb}$. For each physical contig, the MTP BACs were pooled into groups of no more than 20 BACs. These BAC pools were then shotgun sequenced using Illumina paired-end technology. The BAC pool sequence data were first assembled separately for each physical contig using ABySS, totalling $882 \mathrm{Mb}$ in 74,572 contigs. The BAC pool-based contigs provided the starting point for integrating the various datasets using Gydle software (Philippe Rigault, Gydle Inc., Quebec City, Canada, https://www.gydle.com/bioinformatics; [34, 35]). An initial multiple alignment was produced using the Nuclear software (Gydle Inc.) as part of the hybrid assembly of the available datasets. Reprocessing of BAC pool assemblies identified BAC ends and removed low-quality reads, and thus allowed BAC clones to be identified that were not true components of the respective pools. Vision software (Gydle Inc.) was used to visualize assemblies in a semi-manual curation process with assembly metrics calculated using Perl, $\mathrm{R}$ and Shell scripts. An iterative process provided the basis for integrating extensive mate-pair data, Bionano data and KeyGene Whole Genome Profiling (WGP ${ }^{\mathrm{mw}}$ ) tags [1]. The three stages can be summarized as (1) integrating the BAC pool mapping and sequencing data with multiple mate-pair datasets (see also Additional file 1); (2) extending and refining scaffolds based on iterative realignments of the sequence data; and (3) cross-validating the sequence assembly with physical mapping data to link scaffolds with physical contigs, identifying missing BACs, contaminations and physical contig errors, and allowing for selected regions to undergo interactive editing and visualization in order to produce a locally finished, manually reviewed sequence. It was possible to connect consecutive BAC pool sequence assemblies using Bionano optical maps generated from flow-sorted Chinese Spring 7AS/7AL telosomic lines with the sequence 
structure visualized by fluorescent labelling of Nt.BspQI nickase (GCTCTTC) sites (details below), to construct 124 scaffolds or "islands" covering $735.1 \mathrm{Mb}$. The 18 largest islands comprised more than $50 \%$ of the total sequence.

The Gydle website (https://www.gydle.com/) provides information on accessing the software as well as the solutions and services provided by the Gydle company. The scale and novelty of this work required not only capabilities that were (and still are) not available in any other product (open source or commercial) but also specific developments to accommodate both the integration of specific data and their vizualization (e.g. Figures $1,2 b$, $3 a$ and $b)$. The Gydle software Nuclear and Vision have been utilized in the analysis of several genomes, including the Eucalypt [34] and wheat genomes [1,35].

\section{BAC library fingerprinting}

The BAC clones 7A BAC MTP were fingerprinted as described in [19]. The use of an ABI3730XL with a more sensitive laser improved fingerprinting resolution and made it possible to reduce the amount of BAC DNA sample for electrophoresis, thus lowering fingerprinting costs. Thus, 0.5-1.2 $\mu \mathrm{g}$ instead of 1.0-2.0 $\mu \mathrm{g}$ of BAC DNA was simultaneously digested with 2.0 units each BamHI, EcoRI, $X b a \mathrm{I}, X h o \mathrm{I}$ and HaeIII (New England Biolabs, Beverly, MA, USA) at $37^{\circ} \mathrm{C}$ for $3 \mathrm{~h}$. DNAs were labelled using the SNapShot kit $(0.4 \mu \mathrm{l}$ of reagent, Applied Biosystems, Foster City, CA, USA) at $65{ }^{\circ} \mathrm{C}$ for $1 \mathrm{~h}$ and precipitated with ethanol. DNAs were dissolved in $9.9 \mu \mathrm{l}$ of Hi-Di formamide, and $0.3 \mu \mathrm{l}$ of Liz1200 size standard was added to each sample. Restriction fragments were sized on the ABI3730XL. Raw outputs from BAC fingerprinting were converted to .gm format using GeneMapper and filtered with Genoprofiler. The resulting files consisted of lists of numbers denoting fragment size for each BAC, added to an offset for each colour: 0 for blue, 10,000 for green, 20,000 for yellow, 30,000 for red.

\section{Sequencing of MTP BACs}

BAC clone DNA was prepared by a standard alkaline lysis mini-prep procedure. BAC clones were grown overnight on $1.2 \mathrm{ml}$ of $2 \mathrm{YT}$ media with chloramphenicol in 96-well culture plates. Plates were spun by centrifugation at $2500 \mathrm{~g}$ for $10 \mathrm{~min}$ to pellet cells. Each pellet was resuspended in $400 \mu \mathrm{l}$ of GTE buffer $(0.05 \mathrm{M}$ glucose, $0.01 \mathrm{M}$ ethylenediaminetetraacetic acid (EDTA), $0.025 \mathrm{M}$ Tris $\mathrm{pH}$ 7.4). We transferred $60 \mu \mathrm{l}$ of the resuspended cells to an extraction plate, and $100 \mu \mathrm{l}$ of $\mathrm{NaOH} /$ sodium dodecyl sulphate (SDS) solution $(0.8 \% \mathrm{NaOH}, 1 \% \mathrm{SDS})$ was added to lyse the cells. This solution was neutralized by the addition of $100 \mu \mathrm{l}$ of potassium acetate $(3 \mathrm{M})$ and gently mixed by inversion. Lysates were vacuum-filtered through a Costar 96-well filter plate (0.2- $\mu \mathrm{m}$ GHP membrane) and precipitated by the addition of $110 \mu \mathrm{l}$ isopropanol. The BAC DNA was pelleted by centrifugation at $2500 \mathrm{~g}$ for $15 \mathrm{~min}$. The supernatant was removed, and the pellets washed once with $200 \mu \mathrm{l}$ of ice-cold $70 \%$ ethanol. The pellet was let air-dry for 20-30 min and resuspended in $50 \mu \mathrm{l}$ of water.

\section{Illumina sample preparation and sequencing}

We sheared $100 \mathrm{ng}$ of BAC DNA in $50 \mu \mathrm{l}$ by ultra-sonication using the Covaris E220 instrument settings Duty Factor = $5 \%$, Intensity $=5$, Cycles per burst $=200$, Duration $=55 \mathrm{~s}$, Displayed Power $13 \mathrm{~W}$, temperature $5.5-6.0{ }^{\circ} \mathrm{C}$ (Covaris, Woburn, MA, USA). Samples were processed using the Illumina TruSeq HT DNA sample preparation kit (FC-121-2003) as per the manufacturer's guidelines (Illumina, San Diego, CA, USA). Following ligation of adapters, a "double-sided" solid phase reversible immobilization (SPRI) size selection was performed to select for library fragments with a median size of 550600 bp. Libraries were assessed by gel electrophoresis (Agilent D1000 ScreenTape Assay, Cat. Nos. 5067-5582 and 5067-5583; Agilent, Santa Clara, CA, USA) and quantified by quantitative polymerase chain reaction (qPCR) (KAPA Library Quantification Kits for Illumina, Cat. No. KK4835). Sequencing was performed on the HiSeq 2500 system using TruSeq Rapid PE Cluster Kit HS (Cat. No. PE-402-4001) and TruSeq Rapid SBS Kit HS (Cat. Nos. FC-402-4001 and FC-402-4002).

The minimum tiling paths (MTPs) of contigs from the first version of the physical assembly were used to define pools of BACs for sequencing. Large pools (more than 20 BACs in the MTP) were split into multiple pools. We fragmented $100 \mathrm{ng}$ of pooled BAC DNA by ultra-sonication (Covaris E200), and DNA libraries with an insert size of 450 bp were prepared using the Illumina TruSeq DNA HT Sample Preparation Kit. The size of each library was validated using the DNA 1000 ScreenTape (Agilent) and quantified by qPCR before normalization and pooling. We sequenced $96 \mathrm{BAC}$ pool libraries in one lane of the Illumina HiSeq 2500 in rapid mode with $2 \times 150$ bp paired-end reads.

\section{Read filtering and removal of bacterial sequences}

All available Escherichia coli genome sequences in NCBI were used to remove non-wheat sequences because some sequences were found from unexpected strains. The reads underwent quality control to remove contaminating sequences and poor-quality reads before running assembly scripts.

\section{Mate-pair sequencing}

Amplified DNA was produced from the DNA isolated from flow-sorted 7AS and 7AL telosomic chromosome arms using flow-sorted chromosomes treated with 
proteinase $\mathrm{K}$ and amplified using Phi29 multiple displacement amplification (MDA). Overnight amplification in a 20- $\mu$ l reaction produced 3.7-5.7 $\mu$ g DNA with a majority of products between 5 and $30 \mathrm{~Kb}$. This amplified DNA was then processed to remove nicks and single-stranded DNA before carrying out the Nextera Mate Pair/HiSeq System (following manufacturer's instructions) for generating a high coverage of mate-pair sequence information. The libraries covered 200-5000 bp.

\section{PacBio sequencing}

Short-read data and PacBio sequencing of a single BAC (7AS-066B03) followed protocols provided by the technology provider.

\section{Bionano view of genome sequence}

A total of 2.8 million of each of the 7A arms, corresponding to $1.14 \mu \mathrm{g}$ DNA, were purified by flow cytometric sorting as described above with purities of $80 \%$ and $86 \%$ for the 7AS and 7AL arms, respectively. Chromosome arm DNA was used to construct Bionano maps following the protocol of Staňková et al. [17]. Based on the frequency of recognition sites in the survey sequences of 7A arms (IWGSC, 2014), Nt.BspQI nickase (GCTCTTC recognition site) with an estimated frequency of 11 sites/100 Kb was selected for DNA labelling. Chromosome arm DNA samples were labelled at nicking sites with Alexa546-dUTP fluorochrome, and their DNA was stained with YOYO and analysed on the Irys platform (Bionano Genomics, San Diego, CA, USA). Bionano maps of 7AS and 7AL, assembled de novo using molecules longer than $150 \mathrm{~kb}$, exhibited a coverage of $192 \times(79 \mathrm{~Gb})$ and $238 \times(97 \mathrm{~Gb})$, respectively.

De novo assembly of Bionano maps was performed by a pairwise comparison of all single molecules and graph building [36]. A $p$-value threshold of $1 \mathrm{e}^{-10}$ was used during the pairwise assembly, $1 \mathrm{e}^{-10}$ for extension and refinement steps, and $1 \mathrm{e}^{-15}$ for final refinement. The use of Bionano data in the 7A assembly is a significant advance over the work of Staňková et al. [17], as the Gydle software performs a scalable and true hybrid optical/sequence assembly enabling local sequence resolution (e.g. gaps, tandem repeats) based on systematic comparisons of distances in optical and sequence space, as well as map validation using molecule data.

\section{Linkage disequilibrium analysis}

A diverse spring bread wheat collection $(n=863)$ comprising landraces and elite cultivars was used in this study to understand the haplotype structure and extent of linkage disequilibrium (LD) in the yield QTL region on 7A, coordinates $671,200,000-675,300,000 \mathrm{bp}$. LD values were estimated and visualized using the Haploview software [37], and only common SNPs with high minor allele frequency
(MAF > 0.3) and present within $2000 \mathrm{bp}$ on either side of the gene were included in this analysis. A total of 203 SNPs within 35 gene models (plus 2000 bp on either side) spanning the whole region were identified. We could not detect any common SNPs in the remaining 18 genes in the QTL region. The associations (Fig. 4) were colour coded as follows: bright red D' $=1.0$ and LOD $>2.0$ (high LD); light shades of red indicate $\mathrm{D}^{\prime}<1.0$ and LOD $>2.0$ (low-medium LD); white indicates $\mathrm{D}^{\prime}<1.0$ and LOD $<2.0$ (no LD or complete decay).

\section{Defining the centromere}

To confirm the presence of a large missing CENH3 binding domain in the 7AS di-telosomic stock we aligned the 7AS (SRR697699, SRR697706, SRR697723) and 7AL (SRR697675, SRR697676, SRR697680), 101 bp paired-end Illumina reads generated for the CSS assembly to the chromosome 7A assembly using Nuclear software (Gydle) with filtering for minimum base quality of Phred 20, minimum length per side of 50 and paired reads only, and mapping parameters allowing a single mismatch in an HSP of length 50, a minimum alignment length of $50 \mathrm{bp}$, a sensitivity of 25 and a k of 13 ( 98\% identity). See also Additional files 12 and 13.

\section{Availability of data and material}

The data and material used are as follows:

- Wheat chromosome 7A mate-pair data from flowsorted chromosomes [38]

- IWGSC Wheat Chromosome 7A BACs sequenced in pools based on the physical map minimum tiling path (MTP) with Illumina HiSeq 2500 [39]

- Sequencing of a Chinese Spring wheat with 7EL addition from Thinopyrum elongatum [40]

- Stage 3 Gydle assembly of chromosome 7A in fasta format and Bionano assemblies [41].

\section{Additional files}

\section{Additional file 1: BAC preparation and analysis for physical maps.} (DOCX 16 kb)

Additional file 2: Figures S1-S7. (with legends) for Additional files. (DOCX 5813 kb)

Additional file 3: a Combining the MAGIC 8-way cross 7A and Chinese Spring $\times$ Renan 7A maps $[42,43]$. $\mathbf{b}$ Curated genetic map of 7A for anchoring the genome sequence. (ZIP $112 \mathrm{~kb}$ )

Additional file 4: DNA sequence assembly details. (DOCX 15 kb)

Additional file 5: a IWGSC RefSeq 7A inter-scaffold gaps related to PacBio and Gydle scaffolds. b Gydle island IDs related to Bionano maps and BAC sets. (ZIP $51 \mathrm{~kb}$ )

Additional file 6: IWGSC RefSeq v1.0 7A intra-scaffold gaps related to Gydle scaffolds and contigs plus bridging sequences. (XLSX 398 kb)

Additional file 7: IWGSC RefSeq v1.0 7A gene IDs corrected by manual curation. (XLSX 21 kb) 
Additional file 8: Genome association analyses for variation in grain fructan and yield (grain number) [44-47]. (DOCX $17 \mathrm{~kb}$ )

Additional file 9: 7A QTL analysis and coordinates in RAC875 × Kukri cross. (XLSX $24 \mathrm{~kb}$ )

Additional file 10: Finished 7A yield region assembly BAC sets. (XLSX $10 \mathrm{~kb}$ )

Additional file 11: 7A yield region annotation. (XLSX $20 \mathrm{~kb}$ )

Additional file 12: Rice chromosome 8 centromere synteny analysis with chromosome 7A. (DOCX $14 \mathrm{~kb})$

Additional file 13: Quantification of centromere fluorescence for CEN $\mathrm{H} 3$ antibody in situ locations. (DOCX $14 \mathrm{~kb}$ )

\section{Abbreviations}

Contig: Consensus region of DNA sequence represented by overlapping sequence reads. Can have unresolved bases (N), but no gaps; Gb: Gigabase pairs; Island: Genomic region represented by overlapping sets of DNA sequences (scaffolds), physical entities (optical map or molecule, physical clone) or both; Kb: Kilobase pairs; Mb: Megabase pairs; Scaffold: Consensus region of DNA sequence represented by ordered (but not necessarily oriented) contigs, separated by gaps of known (estimated) length; Superscaffold: A portion of the genome sequence where scaffolds have been ordered and oriented relative to each other

\section{Acknowledgements}

The authors are grateful to DM Appels for her dedication in establishing the early molecular genetic maps for chromosome 7A. Bernd Friebe is acknowledged for his guidance in the cytological studies of 7AS and 7AL. Etienne Paux is acknowledged for his guidance with the genetic and physical map alignments. Part of this work was supported by resources provided by the Pawsey Supercomputing Centre with funding from the Australian Government and the Government of Western Australia. The authors are grateful to Professor Jia Jizeng for discussion in relation to the genome assembly of Aikang 58.

\section{Funding}

Australian Government Department of Industry, Innovation, Science, Research and Tertiary Education (funding agreement ACSRF00542), BioPlatforms Australia (BPA) and Grains Research Development Corporation (agreement UMU00037) are thanked for funding the chromosome 7A project. CSIRO Plant Industry, Australia, funded the establishment of the MAGIC molecular genetic map. Agriculture Victoria Research funded bioinformatics capacity and infrastructure. The CENH3 antibody/cytological studies were supported by National Science Foundation (NSF) grant contract 1338897. The work of FC was supported by the INB ("Instituto National de Bioinformatica") Project PT13/0001/0021 (ISCIII -FEDER). Chromosome flowsorting, construction of BAC libraries and Bionano maps were partially supported by Czech Ministry of Education Youth and Sports (award LO1204 from the National Program of Sustainability). The RAC875/Kukri data were supported by the Australian Research Council Industrial Transformation Research Hub for Genetic diversity and molecular breeding for wheat in a hot and dry climate (project number IH130200027) and Australian Grain Technologies.

\section{Availability of data and materials}

The new genome sequence data are submitted to the IWGSC Data Repository hosted at URGI: https://wheat-urgi.versailles.inra.fr/Seq-Repository

\section{Authors' contributions}

GK-G, PR, JT contributed equally to experimental design, data analysis and interpretation/writing of manuscript; $R P, M H, K F, R A$ genome analyses and interpretation; ZF, AK data analysis and physical map construction; EH, CC, JT MAGIC map construction; MA rice-wheat phylogenomic; AS, DK, mate-pair libraries; PS. BD, FC, PL, Chinese Spring $x$ Renan molecular genetic map and annotation of genome sequence; NW-H, UB, PE, DF, AJ analysis of gene space and QTL, SB, M-AN, development of Bionano alignments and tools; JD, HŠ, JŠ, HT, flow sorting of chromosomes, BAC library construction and Bionano maps; $\mathrm{M}-\mathrm{CL}$ fingerprinting of BAC library; FC, MP gene annotation; DC, ZR, AK58 genome assembly, JN-P, genome assembly; DI in-house software development; IWGSC, genome assembly network, D-HK, CENH3 antibody cytology; RA, planning of experiments and writing of manuscript. All authors have read and approved the final manuscript.

\section{Competing interests}

$\mathrm{PR}, \mathrm{SB}$, and M-AN have competing commercial interests as employees and stockholders of Gydle, which is a commercial company that provides bioinformatics analysis software and services. This does not alter the authors' adherence to all of the Genome Biology policies on sharing data and materials. The remaining authors declare that they have no competing interests.

\section{Publisher's Note}

Springer Nature remains neutral with regard to jurisdictional claims in published maps and institutional affiliations.

\section{Author details \\ ${ }^{1}$ Agriculture Victoria Research, Department of Economic Development, Jobs, Transport and Resources, AgriBio, Bundoora, VIC 3083, Australia. ${ }^{2}$ GYDLE, 1135 Grande Allée Ouest, Suite 220, Québec, QC G1S 1E7, Canada. ${ }^{3}$ Center for Organismal Studies (COS), University of Heidelberg, Im Neuenheimer Feld 345, 69120 Heidelberg, Germany. ${ }^{4}$ Institute of Evolution, University of Haifa, Haifa, Israel. ${ }^{5}$ CSIRO-Plant Industry, Black Mountain, Canberra, ACT 2601, Australia. ${ }^{6}$ King Abdullah University of Science and Technology, Desert Agriculture Initiative, Thuwal, Saudi Arabia. ${ }^{7}$ Global Institute of Food Security, University of Saskatchewan, 110 Gymnasium Place, Saskatoon, SK, Canada. ${ }^{8}$ INRA UMR1095 Genetics, Diversity and Ecophysiology of Cereals, 5 chemin de Beaulieu, 63039 Clermont-Ferrand, France. ${ }^{9}$ School of Agriculture, Food and Wine, University of Adelaide, Urrbrae, South Australia 5064, Australia. ${ }^{10}$ Veterinary and Agriculture, Murdoch University, 90 South St, Murdoch, Western Australia 6150, Australia. ${ }^{11}$ Institute of Experimental Botany, Centre of the Region Haná for Biotechnological and Agricultural Research, Slechtitelu 31, CZ-78371 Olomouc, Czech Republic. ${ }^{12}$ UC Davis Plant Sciences, Plant Genetics and Bioinformatics, 258A Hunt Hall, Davis, CA 95616, USA. ${ }^{13}$ Bioinformatics and Genomics Program, Centre for Genomic Regulation (CRG) and Universitat Pompeu Fabra (UPF), 88 Dr. Aiguader, 08003 Barcelona, Spain. ${ }^{14}$ Plant Genome and Systems Biology, Helmholtz Center, Munich, 85764 Neuherberg, Germany. ${ }^{15}$ Level Five Co. Ltd. GYB Akihabara, Kanda-Sudacho 2-25, Chiyoda-ku, Tokyo 101-0041, Japan. ${ }^{16}$ International Wheat Genome Sequencing Consortium, 2841 NE Marywood Ct, Lee's Summit, MO 64086, USA. ${ }^{17}$ Wheat Genetics Resource Center and Department of Plant Pathology, Kansas State University, Manhattan, KS 66506, USA. ${ }^{18}$ Australian Genome Research Facility, Suite 219, 55 Flemington Road, North Melbourne, VIC 3051, Australia. ${ }^{19}$ Henan Agricultural University, Zhengzhou, China. ${ }^{20}$ Henan Institute of Science and Technology, Zhengzhou, China. ${ }^{21}$ National Research Council of Canada, University of Saskatchewan, 110 Gymnasium Place, Saskatoon, SK, Canada.}

Received: 18 February 2018 Accepted: 9 July 2018

Published online: 17 August 2018

\section{References}

1. The International Wheat Genome Sequencing Conosrtium. Shifting the limits in wheat research and breeding through a fully annotated and anchored reference genome sequence. Science. 2018. https://doi.org/10. 1126/science.aar7191.

2. The International Wheat Genome Sequencing Consortium. A chromosomebased draft sequence of the hexaploid bread wheat (Triticum aestivum) genome. Science. 2014;345:1251788.

3. Clavijo BP, Kettleborough G, Heavens D, Chapman H, Lipscombe J, Barker T, Lu F-H, McKenzie N, Raats D, Ramirez-Gonzalez RH, Coince A, Peel N, Percival-Alwyn L, Duncan O, Trösch J, Yu G, Bolser DM, Namaati G, Kerhornou A, Spannagl M, Gundlach H, Haberer G, Davey RP, Fosker C, Di Palma FD, Phillips AL, Millar AH, Kersey PJ, Uauy C, Krasileva KW, Swarbreck D, Bevan MW, Clark MD. An improved assembly and annotation of the allohexaploid wheat genome identifies complete families of agronomic genes and provides genomic evidence for chromosomal translocations. Genome Res. 2017;27:885-96.

4. Zimin AV, Puiu D, Hall R, Kingan S, Salzberg SL (2017), The first nearcomplete assembly of the hexaploid bread wheat genome, Triticum aestivum. GigaScience; $6: 1-7$.

5. Eversole K, Rogers J, Keller B, Appels R, Feuillet C. Sequencing and assembly of the wheat genome. In: Achieving sustainable cultivation of wheat, 
Part 1, Chap. 2. Cambridge: Burleigh-Dodds Science Publishing; 2017. https://doi.org/10.19103/AS.2016.0004.04.

6. Cole CG, McCann OT, Collins JE, Oliver K, Willey D, Gribble SM, Yang F, McLaren K, Rogers J, Ning Z, Beare DM, Dunham I. Finishing the finished human chromosome 22 sequence. Genome Biol. 2008;9:R78. https://doi.org/ 10.1186/gb-2008-9-5-r78

7. Fonville NC, Velmurugan KR, Tae H, Vaksman Z, LJ Ml, Garner HR. Genomic leftovers: identifying novel microsatellites, overrepresented motifs and functional elements in the human genome. Sci Rep. 2016;6:27722. https://doi.org/10.1038/srep27722.

8. Dubcovsky J, Drorak J. Genome plasticity a key factor in the success of polyploid wheat under domestication. Science. 2007;316:1862-6.

9. Shiferaw B, Smale M, Braun H-J, Duveiller E, Reynolds M, Muricho G. Crops that feed the world 10. Past successes and future challenges to the role played by wheat in global food security. Food Security. 2013;5:291-317.

10. Feuillet C, Stein N, Rossini L, Praud S, Mayer K, Schulman A, Eversole K, Appels R. Integrating cereal genomics to support innovation in the Triticeae. Funct Integr Genomics. 2012;12:573-83. https://doi.org/10. 1007/s10142-012-0300-5.

11. Gegas VC, Nazari A, Griffiths S, Simmonds J, Fish L, Orford S, Sayers L, Doonan JH, Snape JW. A genetic framework for kernel size and shape variation in wheat. Plant Cell. 2010;22:1046-56.

12. Boeven $\mathrm{PHG}$, Longin $\mathrm{CFH}$, Leiser $\mathrm{WL}$, Kollers $\mathrm{S}$, Ebmeyer $\mathrm{E}$, Würschum $\mathrm{T}$. Genetic architecture of male floral traits required for hybrid wheat breeding. Theor Appl Genet. 2016;129:2343-57.

13. Wittkop B, Nagorny S, Snowdon R, Friedt W. Breeding progress in wheat: dissecting the components of grain yield. Proc 13th Int Wheat Genetics Symp April 23-28, Tulln, Austria: editors: Buerstmayr H, Lang-Mladek C, Steiner B, Michel S, Maria Buerstmayr M, Lemmens M, Vollmann J, Grausgruber $\mathrm{H}$.

14. Ma Z, Zhao D, Zhang C, Zhang Z, Xue X, Lin F, Kong Z, Tian D, Luo Q. Molecular genetic analysis of five spike-related traits in wheat using RIL and immortalized F2 populations. Mol. Genet. Genomics. 2017;277:31-42. https://doi.org/10.1007/s00438-006-0166-0

15. Su Z, Jin S, Lu Y, Zhang G, Chao S, Bai G. Single nucleotide polymorphism tightly linked to a major QTL on chromosome 7A for both kernel length and kernel weight in wheat. Mol Breeding. 2016;36:15. https://doi.org/10. 1007/s11032-016-0436-4

16. Huynh B-L, Mather DE, Schreiber AW, Toubia J, Baumann U, Shoaei Z, Stein N, Ariyadasa R, Stangoulis JCR, Edwards J, Shirley N, Langridge P, Fleury D. Clusters of genes encoding fructan biosynthesizing enzymes in wheat and barley. Plant Mol Biol. 2012;80:299-314.

17. Staňková H, Hastie AR, Chan S, Vrána J, Tulpová Z, Kubaláková M, Visendi P, Hayashi S, Luo M, Batley J, Edwards D, Doležel J, Šimková H. Bionano genome mapping of individual chromosomes supports physical mapping and sequence assembly in complex plant genomes. Plant Biotechnol J. 2016:14:1523-31.

18. Pingault L, Choulet F, Alberti A, Glover N, Wincker P, Feuillet C, Paux E. Deep transcriptome sequencing provides new insights into the structural and functional organization of the wheat genome. Genome Biol. 2015;16:29.

19. Luo MC, Thomas C, You FM, Hsiao J, Ouyang S, Buell CR, Malandro M, McGuire PE, Anderson OD, Dvorak J. High-throughput fingerprinting of bacterial artificial chromosomes using the snapshot labeling kit and sizing of restriction fragments by capillary electrophoresis. Genomics. 2003:82:378-89.

20. Frenkel Z, Paux E, Mester D, Feuillet C, Korol A. LTC: a novel algorithm to improve the efficiency of contig assembly for physical mapping in complex genomes. BMC Bioinformatics. 2010;11:584. https://doi.org/10.1186/14712105-11-584.

21. Simpson JT, Wong K, Jackman SD, Schein JE, SJM J, Birol I. ABySS: a paralle assembler for short read sequence data. Genome Res. 2009;19:1117-23. https://doi.org/10.1101/gr.089532.108.

22. Benjamini $Y$, Speed TP. Summarizing and correcting the GC content bias in high-throughput sequencing. Nucleic Acids Res. 2012;40:e72. https://doi.org/10.1093/nar/gks001.

23. Quarrie SA, Pekic-Quarrie S, Radosevic R, Rancic D, Kaminska A, Barnes JD, Leverington M, Ceoloni C, Dodig D. Dissecting a wheat QTL for yield present in a range of environments: from the QTL to candidate genes. J Exp Bot. 2006;57:2627-37.

24. Wang G, Zhang X, Jin WJ. An overview of plant centromeres. Genet Genomics. 2009;36:529-37. https://doi.org/10.1016/S1673-8527(08)60144-7.
25. Comai L, Shamoni M, Mohan M, Marimuthu PA. Plant centromeres. Current Opinion in Plant Biology. 2017;36:158-67. doi.org/10.1016/j.pbi.2017.03.003

26. Yan $H$, Talbert PB, Lee HR, Jett J, Henikoff S, Chen F, Jiang J. Intergenic locations of rice centromeric chromatin. PLoS Biol. 2008;6:e286. https://doi.org/10.1371/journal.pbio.0060286.

27. Guo X, Su H, Shi Q, Fu S, Wang J, Zhang X, Hu Z, Han F. De novo centromere formation and centromeric sequence expansion in wheat and its wide hybrids. PLoS Genet. 2016;12:e1005997. https://doi.org/10.1371/ journal.pgen.1005997.

28. Koo DH, Sehgal SK, Friebe B, Gill BS. Structure and stability of telocentric chromosomes in wheat. PLoS One. 2015;10:e0137747. https://doi.org/10. 1371/journal.pone.0137747.

29. Chapman JA, Mascher M, Buluç A, Barry K, Georganas E, Session A, Strnadova V, Jenkins J, Sehgal S, Oliker L, Schmutz J, Yelick KA, Scholz U, Waugh R, Poland JA, Muehlbauer GJ, Stein N, Rokhsar DS. A whole-genome shotgun approach for assembling and anchoring the hexaploid bread wheat genome. Genome Biol. 2015;16:26.

30. Pollock CJ, Cairns AJ. Fructan metabolism in grasses and cereals. Annu Rev Plant Physiol Plant Mol Biol. 1991;42:77-101. https://doi.org/10.1146/ annurev.pp.42.060191.000453.

31. Dawe RK. Meiotic chromosome organization and segregation in plants. Annu Rev Physiol Plant Mol Biol. 1998;49:371-95.

32. Kishii $\mathrm{M}$, Nagaki $\mathrm{K}$, Tsujimoto $\mathrm{H}$. A tandem repetitive sequence located in the centromeric region of common wheat (Triticum aestivum) chromosomes. Chromosom Res. 2001;9:417-28.

33. Malik HS, Henikoff S. Conflict begets complexity: the evolution of centromeres. Curr Opin Genet Dev. 2002;12:711-8.

34. Myburg AA, Grattapaglia D, Tuskan GA, Hellsten U, Hayes RD, Grimwood J, Jenkins J, Lindquist E, Tice H, Bauer D, Goodstein DM, Dubchak I, Poliakov A, Mizrachi E, Kullan AR, Hussey SG, Pinard D, van der Merwe K, Singh P, van Jaarsveld I, Silva-Junior OB, Togawa RC, Pappas MR, Faria DA, Sansaloni CP, Petroli CD, Yang X, Ranjan P, Tschaplinski TJ, Ye CY, Li T, Sterck L, Vanneste K, Murat F, Soler M, Clemente HS, Saidi N, Cassan-Wang H, Dunand C, Hefer CA, Bornberg-Bauer E, Kersting AR, Vining K, Amarasinghe V, Ranik M, Naithani S, Elser J, Boyd AE, Liston A, Spatafora JW, Dharmwardhana P, Raja R, Sullivan C, Romanel E, Alves-Ferreira M, Külheim C, Foley W, Carocha V, Paiva J, Kudrna D, Brommonschenkel SH, Pasquali G, Byrne M, Rigault P, Tibbits J, Spokevicius A, Jones RC, Steane DA, Vaillancourt RE, Potts BM, Joubert F, Barry K, Pappas GJ, Strauss SH, Jaiswal P, Grima-Pettenati J, Salse J, Van de Peer Y, Rokhsar DS, Schmutz J. The genome of Eucalyptus grandis. Nature. 2014:510:356-62. https://doi.org/10.1038/nature13308.

35. Barrero JM, Cavanagh C, Verbyla KL, Tibbits JF, Verbyla AP, Huang BE, Rosewarne GM, Stephen S, Wang P, Whan A, Rigault P, Hayden MJ, Gubler F. Transcriptomic analysis of wheat near-isogenic lines identifies PM19-A1 and A2 as candidates for a major dormancy QTL.Genome Biol. 2015;16:93. https://doi.org/10.1186/s13059-015-0665-6.

36. Cao H, Hastie AR, Cao D, Lam ET, Sun Y, Huang H, et al. Rapid detection of structural variation in a human genome using nanochannel-based genome mapping technology. GigaScience. 2014;3. https://doi.org/10.1186/2047217X-3-34.

37. Barrett JC, Fry B, Maller J, Daly MJ. Haploview: analysis and visualization of LD and haplotype maps. Bioinformatics. 2005;21:263-5. PubMed ID: 15297300.

38. Wheat chromosome 7A mate-pair data from flow-sorted chromosomes. 2018. https://www.ebi.ac.uk/ena/data/view/PRJEB26335.

39. IWGSC Wheat chromosome 7A BACs sequenced in pools based on the physical map minimum tiling path (MTP) with Illumina HiSeq 2500. 2018. https://www.ebi.ac.uk/ena/data/view/PRJEB26029.

40. Sequencing of a Chinese spring wheat with 7EL addition from Thinopyrum elongatum. 2018. https://www.ebi.ac.uk/ena/data/view/PRJNA450404.

41. Stage 3 Gydle assembly of chromosome 7A and Bionano assemblies. 2018. https://urgi.versailles.inra.fr/download/iwgsc/7A.

42. Huang BE, George AW, Forrest KL, Kilian A, Hayden MJ, Morell MK, Cavanagh CR. A multiparent advanced generation inter-cross population for genetic analysis in wheat. Plant Biotechnol J. 2012;10:826-39. https://doi.org/10.1111/j.1467-7652.2012.00702.x.

43. Shah $R$, Cavanagh CR, Huang BE. Computationally efficient map construction in the presence of segregation distortion. Theor Appl Genet. 2014;127:2585-97.

44. Sehgal D, Autrique E, Singh R, Ellis M, Singh S, Dreisigacker S. Identification of genomic regions for grain yield and yield stability and their epistatic interactions. Sci Rep. 2017;7:41578. https://doi.org/10.1038/srep41578. 
45. Taylor J, Butler D. R Package ASMap: efficient genetic linkage map, construction and diagnosis. J Stat Softw 2017. https://doi.org/10.18637/jss.V079.106.

46. Bennett D, Reynolds M, Mullan D, Izanloo A, Kuchel H, Langridge P, Schnurbusch T. Detection of two major grain yield QTL in bread wheat (Triticum aestivum L.) under heat, drought and high yield potential environments. Theor Appl Genet. 2012;125:1473-85. https://doi.org/10.1007/s00122-012-1927-2.

47. Taylor J, Verbyla A. R Package wgaim: QTL analysis in bi-parental populations using linear mixed models. J Stat Softw. 2011:40:1-18. http://www.jstatsoft.org/v40/i07/

48. Ma M, Wang Q, Li Z, Cheng H, Li Z, Liu X, Song W, Appels R, Zhao H. Expression of TaCYP78A3, a gene encoding cytochrome P450 CYP78A3 protein in wheat (Triticum aestivum L.), affects seed size. Plant J. 2015;83: 312-25. https://doi.org/10.1111/tpj.12896.

Ready to submit your research? Choose BMC and benefit from:

- fast, convenient online submission

- thorough peer review by experienced researchers in your field

- rapid publication on acceptance

- support for research data, including large and complex data types

- gold Open Access which fosters wider collaboration and increased citations

- maximum visibility for your research: over $100 \mathrm{M}$ website views per year

At BMC, research is always in progress.

Learn more biomedcentral.com/submissions 